\title{
On the Model and Invariant Subspaces for Pairs of Commuting Isometries
}

\author{
Zbigniew Burdak@
}

\begin{abstract}
The paper is devoted to a model and joint invariant subspaces under a pair of commuting isometries. A certain class of pairs of commuting isometries is defined. We give a model for such pairs and show that an arbitrary pair of commuting isometries has a minimal extension to a pair in the defined class. Subsequently we investigate a model for a general commuting pair of isometries via joint invariant subspaces of this extension. As an application operators of multiplication by independent variables on the Hardy space over the torus are extended to a pair in the defined class and joint invariant subspaces of the extension are described.
\end{abstract}

Mathematics Subject Classification. Primary 47A15; Secondary 47A20.

Keywords. Invariant subspaces, Beurling theorem, Multiplication operator over bi-disk, Hardy space, Isometries.

\section{Introduction}

The Wold decomposition [18], precisely the abstract version of Halmos [6], decomposes an isometry between a unitary operator and a unilateral shift. Unitary operators are described by the spectral theorem while the result of Beurling [2] and the following extensions by Lax [8] and Halmos [6] give the model for a unilateral shift and its invariant subspaces. A single isometry is fully characterized by these results.

Neither the Wold-Halmos decomposition nor the Beurling's result generalize to pairs of isometries fully. The most natural extension of Wold's result to a pair of isometries is a quaternary decomposition among: a pair of unitary operators, a pair of unilateral shifts and two mixed pairs. A general pair of commuting isometries may not admit such a decomposition. The example of pairs satisfying it are, by the result of Słociński [17], pairs of doubly commuting isometries (i.e., operators commute and each of them commute

This research was financed by the Ministry of Science and Higher Education of the Republic of Poland. 
with the adjoint of the other operator). Hereof, the above quaternary decomposition is called the Wold-Słociński decomposition. There are non doubly commuting pairs that admit the Wold-Słociński decomposition. However, an arbitrary pair of commuting isometries decomposes with an additional fifth summand. A precise description of the additional summand remains a subject of research.

Another unknown part of a model for pairs of commuting isometries concerns pairs of unilateral shifts. A two dimensional Beurling type result provides a model for a pair of doubly commuting unilateral shifts. Moreover, according to examples of Rudin [11], the Beurling type result for pairs of doubly commuting isometries does not describe all joint invariant subspaces, but only such where the operators continue to doubly commute - see [9]. There are, however, other invariant subspaces, some of them are described by inner functions used in an extended way (cf. $[4,5,7,16]$ ). Even less is known about commuting, non doubly commuting unilateral shifts. It is noteworthy that the results involving double commutativity mentioned above extend to $n$ tuples (cf. $[14,15])$. For a general $n$ - tuple of commuting isometries probably the best known model is the Berger-Coburn-Lebow result in [1] based on the Beurling-Lax-Halmos model for the product of the $n$-tuple.

Let $\mathcal{B}(H)$ denote the algebra of bounded, linear operators on a separable Hilbert space $H$. A subspace $H_{0} \subset H$ is a closed linear manifold, $\overline{\operatorname{span}}\{\ldots\}$ denotes the closure of a linear manifold spanned by vectors or subspaces listed in brackets and $P_{H_{0}}$ denotes an orthogonal projection onto $H_{0}$ (i.e., $P_{H_{0}}^{2}=$ $P_{H_{0}}=P_{H_{0}}^{*}$ ). Whenever a minimal extension is mentioned, the minimality refers to a space where the extension operate, as a minimal element of a chain with the inclusion order. Denote by $\operatorname{Lat}\left(V_{1}\right)$, Lat $\left(V_{2}\right), \operatorname{Lat}\left(V_{1}, V_{2}\right)$ lattices of (joint) invariant subspaces under isometries $V_{1}, V_{2} \in \mathcal{B}(H)$. Let us recall Wold-Halmos decomposition theorem $[6,18]$.

Theorem 1.1. (Wold-Halmos) Let $V \in \mathcal{B}(H)$ be an isometry. There is a unique decomposition of $H$ between orthogonal, reducing under $V$ subspaces $H_{u}, H_{s}$, such that $\left.V\right|_{H_{u}}$ is a unitary operator and $\left.V\right|_{H_{s}}$ is a unilateral shift. Moreover,

$$
H_{u}=\bigcap_{n \geq 0} V^{n} H, \quad H_{s}=\bigoplus_{n \geq 0} V^{n}\left(\operatorname{ker} V^{*}\right) .
$$

Let $\mathbb{D} \subset \mathbb{C}$ stands for the unit disk with a boundary $\mathbb{T}$ and $L^{2}(\mathbb{T})$ and $H^{2}(\mathbb{T})$ denote the space of scalar valued, square integrable functions and Hardy space, respectively. The space of square integrable functions valued in a separable Hilbert space $\mathcal{H}$ with the inner product induced by the norm $\|f\|=\left(\int_{\mathbb{T}}\|f(z)\|_{\mathcal{H}}^{2} d z\right)^{1 / 2}$ is unitarily equivalent to $L^{2}(\mathbb{T}) \otimes \mathcal{H}$ and is denoted with this symbol. Similarly $H^{2}(\mathbb{T}) \otimes \mathcal{H}$ denotes the space of analytic, square integrable, $\mathcal{H}$ valued functions. Set $P_{n} \in \mathcal{B}\left(H^{2}(\mathbb{T})\right)$ for a projection onto the subspace $\mathbb{C} z^{n}$, and then $P_{n} \otimes I$ is a projection onto $z^{n} \otimes \mathcal{H}$ for $n \geq$ 0 where $z^{n}$ stands for the monomial $\left\{z \mapsto z^{n}\right\}$ in this context. Further, let $L^{\infty}(\mathbb{T}, \mathcal{B}(\mathcal{H}))$ and $H^{\infty}(\mathbb{T}, \mathcal{B}(\mathcal{H}))$ consist of $\mathcal{B}(\mathcal{H})$ valued functions and analytic functions, respectively, measurable in the sense that $\{z \mapsto \theta(z) x\}$ is a measurable function for any $x \in \mathcal{H}$ and bounded in the essential supremum 
norm. It is a normed algebra with an involution where the operations are defined pointwise. Any function $\theta \in L^{\infty}(\mathbb{T}, \mathcal{B}(\mathcal{H}))$ corresponds to the operator $\hat{\theta} \in \mathcal{B}\left(L^{2}(\mathbb{T}) \otimes \mathcal{H}\right)$ defined as $(\hat{\theta} f)\{z \mapsto \theta(z) f(z)\}$. The operation "^" is an algebra isomorphism onto $\hat{L}:=\left\{\hat{\theta}: \theta \in L^{\infty}(\mathbb{T}, \mathcal{B}(\mathcal{H}))\right\}$ and $\hat{H}:=\{\hat{\theta}$ : $\left.\theta \in H^{\infty}(\mathbb{T}, \mathcal{B}(\mathcal{H}))\right\}$, respectively. Operators of multiplication by independent variable $M_{z} \otimes I \in \mathcal{B}\left(L^{2}(\mathbb{T}) \otimes \mathcal{H}\right)$ and $T_{z} \otimes I \in \mathcal{B}\left(H^{2}(\mathbb{T}) \otimes \mathcal{H}\right)$ are models for a bilateral shift and a unilateral shift, respectively, of multiplicities equal to $\operatorname{dim} \mathcal{H}$. The spaces $\hat{L}, \hat{H}$ are commutants of $M_{z} \otimes I, T_{z} \otimes I$, respectively. A function $\theta \in L^{\infty}(\mathbb{T}, \mathcal{B}(\mathcal{H}))$ is a unimodular function, if $\theta(z)$ is a partial isometry with the same initial space $\mathcal{H}_{0}$ for almost every $z \in \mathbb{T}$ (equivalently $\hat{\theta}$ is a partial isometry with the initial space $\left.L^{2}(\mathbb{T}) \otimes \mathcal{H}_{0}\right)$. If, additionally, $\theta \in H^{\infty}(\mathbb{T}, \mathcal{B}(\mathcal{H}))$, then it is called an inner function. Inner functions describe all subspaces invariant under a unilateral shift by the following result $[6$, Theorem 3].

Theorem 1.2. (Beurling-Lax-Halmos) Subspaces invariant under the operator $T_{z} \otimes I \in \mathcal{B}\left(H^{2}(\mathbb{T}) \otimes \mathcal{H}\right)$ are precisely those of the form $\hat{\theta}\left(H^{2}(\mathbb{T}) \otimes \mathcal{H}\right)$ where $\hat{\theta}$ is an inner function.

Most of the facts recalled above are proved by P. R. Halmos [6], see also $[12,13]$.

Note that $\operatorname{Lat}\left(V_{1}, V_{2}\right)=\operatorname{Lat}\left(V_{1}\right) \cap \operatorname{Lat}\left(V_{2}\right)$. However, the Beurling-LaxHalmos result represents $\operatorname{Lat}\left(V_{i}\right)$ by $\mathcal{B}\left(\operatorname{ker} V_{i}^{*}\right)$ valued inner functions. It makes $\operatorname{Lat}\left(V_{1}\right)$ and $\operatorname{Lat}\left(V_{2}\right)$ difficult to compare since the appropriate inner functions are disjointly valued for $\operatorname{ker} V_{1}^{*} \neq \operatorname{ker} V_{2}^{*}$. The main idea of the paper is to extend an arbitrary pair of commuting isometries $\left(V_{1}, V_{2}\right)$ on a Hilbert space $H$ to a pair $\left(\tilde{V}_{1}, \tilde{V}_{2}\right)$ on a Hilbert space $\tilde{H}$ of a certain form, which allows to investigate both $\operatorname{Lat}\left(\tilde{V}_{1}\right)$ and $\operatorname{Lat}\left(\tilde{V}_{2}\right)$ by inner function valued in the same algebra. Then the initial pair is described by the following relations: $H \in \operatorname{Lat}\left(\tilde{V}_{1}, \tilde{V}_{2}\right)$ and $\operatorname{Lat}\left(V_{1}, V_{2}\right)=\left\{H \cap \mathcal{M}: \mathcal{M} \in \operatorname{Lat}\left(\tilde{V}_{1}, \tilde{V}_{2}\right)\right\} \subset$ $\operatorname{Lat}\left(\tilde{V}_{1}, \tilde{V}_{2}\right)$. Precisely, an arbitrary pair of commuting isometries $\left(V_{1}, V_{2}\right)$ is extended to a pair $\left(U^{k} V^{m}, U^{l} V^{n}\right)$ where $U$ is a unitary operator commuting with an isometry $V$ and $k, l, m, n$ are appropriate nonnegative integers. Then $\operatorname{Lat}\left(U^{k} V^{m}, U^{l} V^{n}\right)$ is investigated via $\operatorname{Lat}(V)$.

In Sect. $2 \operatorname{Lat}\left(V^{m}, V^{n}\right)$ is described for a unilateral shift $V$ and $m, n$ positive integers. In Sect. 3 an arbitrary pair of commuting isometries $\left(V_{1}, V_{2}\right)$ is proved to have a minimal extension to a commuting pair of isometries $\left(\tilde{V}_{1}, \tilde{V}_{2}\right)$ satisfying $\tilde{V}_{1}^{m}=\tilde{U} \tilde{V}_{2}^{n}$, where $\tilde{U}$ is a unitary operator commuting with $\tilde{V}_{1}, \tilde{V}_{2}$ and $m, n$ are positive integers. In Sect. 4 a pair of commuting isometries $\left(V_{1}, V_{2}\right)$ satisfying $V_{1}^{m}=U V_{2}^{n}$, for $m, n$ relatively prime, positive integers, is extended to a pair $\left(\tilde{U}^{k} \tilde{V}^{n}, \tilde{U}^{l} \tilde{V}^{m}\right)$ where $\tilde{V}$ is an isometry, $\tilde{U}$ is a unitary operator commuting with $\tilde{V}$ and $k m-l n=1$. Moreover, the extension is minimal and if $V_{1}, V_{2}$ are unilateral shifts, then $\tilde{V}$ is a unilateral shift. Applications are presented in the last section.

\subsection{Preliminary Results}

Let us remark several technical results which are used throughout the paper. 
By Fuglede-Putnam theorem and [6, Theorem 1] we get:

Remark 1.3. Unitary operators commuting with a unilateral shift $T_{z} \otimes I \in$ $\mathcal{B}\left(H^{2}(\mathbb{T}) \otimes \mathcal{H}\right)$ are precisely those of the form $I \otimes \mathcal{U}$ where $\mathcal{U} \in \mathcal{B}(\mathcal{H})$ is a unitary operator.

We take advantage of the following facts:

Remark 1.4. Let $\left(V_{1}, V_{2}\right)$ be a pair of commuting isometries and $i, j, p \in \mathbb{Z}_{+}$.

1. The operator $V_{2}^{* j} V_{1}^{i}$ commutes with $V_{2}$ if and only if $V_{2}^{* j} V_{1}^{i}$ is an isometry. Then $V_{2}^{* j} V_{1}^{i}$ also commutes with $V_{1}$ and $\left(V_{2}^{* j} V_{1}^{i}\right)^{p}=V_{2}^{* p j} V_{1}^{p i}$.

2. If $i \leq j$ then $\left(I-V_{2}^{i} V_{2}^{* i}\right) V_{1}\left(I-V_{2}^{j} V_{2}^{* j}\right)=\left(I-V_{2}^{i} V_{2}^{* i}\right) V_{1}$ and consequently $\left(\left(I-V_{2} V_{2}^{*}\right) V_{1}\right)^{j}=\left(I-V_{2} V_{2}^{*}\right) V_{1}^{j}$.

Indeed, if $V_{2}^{* j} V_{1}^{i}$ commutes with $V_{2}$, then

$$
\left(V_{2}^{* j} V_{1}^{i}\right)^{*} V_{2}^{* j} V_{1}^{i}=V_{1}^{* i} V_{2}^{j} V_{2}^{* j} V_{1}^{i}=V_{1}^{i} V_{2}^{* j} V_{1}^{i} V_{2}^{j}=V_{1}^{* i} V_{2}^{* j} V_{2}^{j} V_{1}^{i}=I,
$$

so $V_{2}^{* j} V_{1}^{i}$ is an isometry.

For the reverse implication note that if $V_{2}^{* j} V_{1}^{i}$ is an isometry then

$$
\left\|V_{2}^{* j-1} V_{1}^{i} f\right\| \geq\left\|V_{2} V_{2}^{*} V_{2}^{* j-1} V_{1}^{i} f\right\|=\left\|V_{2}^{* j} V_{1}^{i} f\right\|=\|f\| \geq\left\|V_{2}^{* j-1} V_{1}^{i} f\right\| .
$$

Hence $\left\|V_{2} V_{2}^{*} V_{2}^{* j-1} V_{1}^{i} f\right\|=\left\|V_{2}^{* j-1} V_{1}^{i} f\right\|$. Since for any projection $P$, if $\|P x\|$ $=\|x\|$, then $P x=x$ and $V_{2} V_{2}^{*}$ is a projection we get $V_{2} V_{2}^{* j} V_{1}^{i} f=V_{2}^{* j-1} V_{1}^{i} f=$ $V_{2}^{* j} V_{1}^{i} V_{2} f$, so $V_{2}^{* j} V_{1}^{i}$ commutes with $V_{2}$.

If $V_{2}^{* j} V_{1}^{i}$ commutes with $V_{2}$, then

$V_{1} V_{2}^{* j} V_{1}^{i}=V_{2}^{* j} V_{2}^{j} V_{1} V_{2}^{* j} V_{1}^{i}=V_{2}^{* j} V_{1} V_{2}^{* j} V_{1}^{i} V_{2}^{j}=V_{2}^{* j} V_{1} V_{2}^{* j} V_{2}^{j} V_{1}^{i}=V_{2}^{* j} V_{1}^{i} V_{1}$, so $V_{2}^{* j} V_{1}^{i}$ commutes with $V_{1}$.

The condition $\left(V_{2}^{* j} V_{1}^{i}\right)^{p}=V_{2}^{* p j} V_{1}^{p i}$ is a consequence of the commutativity between $V_{2}^{* j} V_{1}^{i}$ and $V_{1}$. We argue by induction, the base step $p=1$ is obvious. The inductive step: if $\left(V_{2}^{* j} V_{1}^{i}\right)^{p}=V_{2}^{* p j} V_{1}^{p i}$, then

$$
\begin{aligned}
\left(V_{2}^{* j} V_{1}^{i}\right)^{p+1} & =V_{2}^{* j} V_{1}^{i}\left(V_{2}^{* j} V_{1}^{i}\right)^{p}=V_{2}^{* j}\left(V_{2}^{* j} V_{1}^{i}\right)^{p} V_{1}^{i} \\
& =V_{2}^{* j} V_{2}^{* p j} V_{1}^{p i} V_{1}^{i}=V_{2}^{*(p+1) j} V_{1}^{(p+1) i} .
\end{aligned}
$$

For the second claim note that

$$
\begin{aligned}
\left(I-V_{2}^{i} V_{2}^{* i}\right) V_{1} f & =\left(I-V_{2}^{i} V_{2}^{* i}\right) V_{1}\left(\left(I-V_{2}^{j} V_{2}^{* j}\right) f+V_{2}^{j} V_{2}^{* j} f\right) \\
& =\left(I-V_{2}^{i} V_{2}^{* i}\right) V_{1}\left(I-V_{2}^{j} V_{2}^{* j}\right) f+\left(I-V_{2}^{i} V_{2}^{* i}\right) V_{2}^{j} V_{1} V_{2}^{* j} f \\
& =\left(I-V_{2}^{i} V_{2}^{* i}\right) V_{1}\left(I-V_{2}^{j} V_{2}^{* j}\right) f .
\end{aligned}
$$

The last remark concerns relatively prime numbers.

Remark 1.5. If $m, n$ are relatively prime, positive integers, then:

- for any $i=1, \ldots, n-1$ there are unique integers $k, l$ such that $0<k<n$, $0 \leq l<m$ and $i=k m-l n$,

- the set $A=\mathbb{Z}_{+} \backslash\left\{k m+l n: k, l \in \mathbb{Z}_{+} \cup\{0\}\right\}$ is bounded and $m n-m-n$ is the maximal element of $A$. 
Since $m, n$ are relatively prime, the remainders of division of $m, 2 m, \ldots, n m$ by $n$ are pairwise different. Hence they form a set of cardinality $n$, so the whole set $\{0, \ldots, n-1\}$. Excluding $n m$ and the corresponding reminder 0 we get that for any $i \in\{1, \ldots, n-1\}$ there is a unique $0<k<n$ such that $k m=l n+i$ for some nonnegative integer $l$. Hence $l$ is also unique and $0 \leq l<m$.

For the second claim, denote an integer greater than $m n-m-n$ as $m n-m-n+p n+i$ where $p$ is a nonnegative integer and $i \in\{0, \ldots, n-1\}$. By the first claim $i=k m-l n$ for the appropriate $k, l$ and $m n-m-n+p n+i=$ $m n-m-n+p n+k m-l n=(m-1+p-l) n+(k-1) m$. Note that $0<k$ and $l<m$ yield $m+p-1-l, k-1 \in \mathbb{Z}_{+} \cup\{0\}$. Thus the maximal element of $A$ is at most $m n-m-n$. On the other hand, if $m n-m-n \notin A$, then $m n-m-n=k m+l n$, and so $m n=(k+1) m+(l+1) n$. Since $m, n$ are relatively prime, the last equality implies $k+1=p n,(l+1)=q m$ for positive integers $p, q$. This leads to a contradiction: $m n=(k+1) m+(l+1) n=(p+q) m n>m n$ as $p+q \geq 2$.

\section{Subspaces Invariant Under a Pair of Powers of a Unilateral Shift}

In the section we describe $\operatorname{Lat}\left(V^{m}, V^{n}\right)$ where $V$ is a unilateral shift. The main tool is Beurling-Lax-Halmos characterization of Lat $(V)$. Since invariant subspaces of any unilateral shift are described by this result, we may assume $m, n$ to be relatively prime, for if not, we replace $V$ by $V^{G C D(m, n)}$.

We start with an obvious observation that $\operatorname{Lat}(V) \subset \operatorname{Lat}\left(V^{m}, V^{n}\right)$ but there is no equality in general.

Example 2.1. Let $V \in L(H)$ be a unilateral shift and $\mathcal{M} \in \operatorname{Lat}(V)$. By [6] $\mathcal{M} \ominus V \mathcal{M}$ is a wandering subspace for $\left.V\right|_{\mathcal{M}}$ and $\mathcal{M}=\bigoplus_{n>0} V^{n}(\mathcal{M} \ominus V \mathcal{M})$. Consider $\mathcal{M}_{0}=\mathcal{M} \ominus V(\mathcal{M} \ominus V \mathcal{M})=\bigoplus_{\substack{n \geq 0 \\ n \neq 1}} V^{n}(\mathcal{M} \ominus V \mathcal{M})$. Then $\mathcal{M}_{0} \notin$ Lat $(V)$ but $\mathcal{M}_{0} \in \operatorname{Lat}\left(V^{m}, V^{n}\right)$ provided neither of $m, n$ equals 1.

Obviously, if any of $m, n$ equals 1 , then $\operatorname{Lat}\left(V^{m}, V^{n}\right)=\operatorname{Lat}(V)$.

Corollary 2.2. Let $V$ be a unilateral shift. Then $\operatorname{Lat}(V) \subset \operatorname{Lat}\left(V^{m}, V^{n}\right)$ and they are equal if and only if $m=1$ or $n=1$.

Let us investigate a correspondence between $\operatorname{Lat}(V)$ and $\operatorname{Lat}\left(V^{m}, V^{n}\right)$ more thoroughly.

Lemma 2.3. Let $V \in L(H)$ be a unilateral shift and $m, n$ be relatively prime, positive integers. For any $\mathcal{M} \in \operatorname{Lat}\left(V^{m}, V^{n}\right)$ there is a subspace $\mathcal{N} \in \operatorname{Lat}(V)$ such that $V^{(m-1)(n-1)} \mathcal{N} \subset \mathcal{M} \subset \mathcal{N}$.

Proof. Observe that the minimal, invariant under $V$ subspace containing $\mathcal{M}$ is $\mathcal{N}=\overline{\operatorname{span}}\left\{V^{j} \mathcal{M}: j \geq 0\right\}$. By Remark 1.5 for any $j \geq(m-1)(n-1)$ there are $k_{j}, l_{j} \in \mathbb{Z}_{+}$such that $j=k_{j} m+l_{j} n$. Hence 


$$
\begin{aligned}
V^{(m-1)(n-1)} \mathcal{N} & =\overline{\operatorname{span}}\left\{V^{j} \mathcal{M}: j \geq(m-1)(n-1)\right\} \\
& =\overline{\operatorname{span}}\left\{V^{k_{j} m+l_{j} n} \mathcal{M}: j \geq(m-1)(n-1)\right\} \\
& =\overline{\operatorname{span}}\left\{\left(V^{m}\right)^{k_{j}}\left(V^{n}\right)^{l_{j}} \mathcal{M}: j \geq(m-1)(n-1)\right\} \subset \mathcal{M} .
\end{aligned}
$$

Note that different subspaces in $\operatorname{Lat}\left(V^{m}, V^{n}\right)$ may correspond to the same subspace in $\operatorname{Lat}(V)$ where the correspondence is in the sense of Lemma 2.3. Hence a description of $\operatorname{Lat}\left(V^{m}, V^{n}\right)$ requires an additional invariant.

Theorem 2.4. Let $V$ be a unilateral shift and $m, n$ be relatively prime, positive integers. Subspaces jointly invariant under $\left(V^{m}, V^{n}\right)$ are of the form

$$
V^{(m-1)(n-1)} \mathcal{N} \oplus \mathcal{N}_{0}
$$

where $\mathcal{N} \in \operatorname{Lat}(V)$ and $\mathcal{N}_{0}$ is a subspace of $\mathcal{N} \ominus V^{(m-1)(n-1)} \mathcal{N}$ invariant under $P_{\mathcal{N} \ominus V}(m-1)(n-1) \mathcal{N} V^{\alpha}$ for $\alpha=m, n$.

Proof. Note that $V^{(m-1)(n-1)} \mathcal{N}$ is closed, so it is a subspace.

Let $\mathcal{M}=V^{(m-1)(n-1)} \mathcal{N} \oplus \mathcal{N}_{0}$ where $\mathcal{N}, \mathcal{N}_{0}$ are as in the statement. Then $\mathcal{M} \subset \mathcal{N}$ and $V^{\alpha} x=P_{\mathcal{N}} V^{\alpha} x=P_{V^{(m-1)(n-1)} \mathcal{N}} V^{\alpha} x+P_{\mathcal{N} \ominus V^{(m-1)(n-1)} \mathcal{N}} V^{\alpha} x \in$ $\mathcal{M}$ for $\alpha=m, n$ and $x \in \mathcal{M}$. Hence $\mathcal{M} \in \operatorname{Lat}\left(V^{m}, V^{n}\right)$.

Let $\mathcal{M} \in \operatorname{Lat}\left(V^{m}, V^{n}\right)$ be an arbitrary subspace and $\mathcal{N} \in \operatorname{Lat}(V)$ be a subspace corresponding to $\mathcal{M}$ as in Lemma 2.3. The inclusions $V^{(m-1)(n-1)} \mathcal{N}$

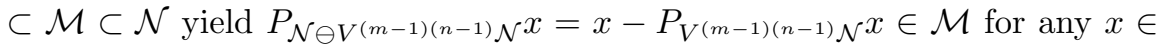
$\mathcal{M}$. Hence $\mathcal{M}$ is invariant under $P_{\mathcal{N} \ominus V^{(m-1)(n-1)} \mathcal{N}}$ (so reducing) and therefore $P_{\mathcal{M}}$ commutes with $P_{\mathcal{N} \ominus V^{(m-1)(n-1)} \mathcal{N}}$. Hence $\mathcal{N}_{0}:=\operatorname{ran}\left(P_{\mathcal{N} \ominus V^{(m-1)(n-1)} \mathcal{N}} P_{\mathcal{M}}\right)$ $\subset \mathcal{M}$ is closed as the product $P_{\mathcal{N} \ominus V^{(m-1)(n-1)} \mathcal{N}} P_{\mathcal{M}}$ is a projection. Note that $\mathcal{M}=\mathcal{N}_{0} \oplus V^{(m-1)(n-1)} \mathcal{N}$ and $V^{\alpha} \mathcal{N}_{0} \subset V^{\alpha} \mathcal{M} \subset \mathcal{M}$ for $\alpha=m, n$. Consequently $P_{\mathcal{N} \ominus V^{(m-1)(n-1)} \mathcal{N}} V^{\alpha} \mathcal{N}_{0} \subset P_{\mathcal{N} \ominus V^{(m-1)(n-1)} \mathcal{N}} \mathcal{M}=\mathcal{N}_{0}$.

Theorem 2.4 describes $\operatorname{Lat}\left(V^{m}, V^{n}\right)$ by Lat $(V)$ characterized by Theo-

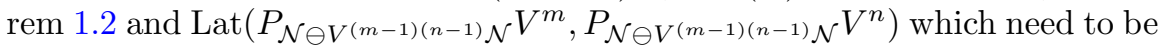
characterized. The latter lattice may be easier to investigate than $\operatorname{Lat}\left(V^{m}, V^{n}\right)$ since the operators act on a smaller space. In particular they act on a finite dimensional space for $V$ of finite multiplicity. The other example where a description of $\operatorname{Lat}\left(P_{\mathcal{N} \ominus V^{(m-1)(n-1)} \mathcal{N}} V^{m}, P_{\mathcal{N} \ominus V^{(m-1)(n-1)} \mathcal{N}} V^{n}\right)$ is easier is the case $m=2, n=3$.

Corollary 2.5. For a unilateral shift $V$ we have

$$
\operatorname{Lat}\left(V^{2}, V^{3}\right)=\left\{V^{2} \mathcal{N} \oplus \mathcal{N}_{0}: \mathcal{N} \in \operatorname{Lat}(V), \mathcal{N}_{0} \subset \mathcal{N} \ominus V^{2} \mathcal{N}\right\}
$$

Proof. Since $\left.P_{\mathcal{N} \ominus V^{2} \mathcal{N}} V^{\alpha}\right|_{\mathcal{N}}=0$ for $\alpha=2,3$, any subspace $\mathcal{N}_{0} \subset \mathcal{N} \ominus V^{2} \mathcal{N}$ satisfies the assumptions of Theorem 2.4.

Corollary 2.5 and Theorem 1.2 provide the full description of $\operatorname{Lat}\left(V^{2}\right.$, $\left.V^{3}\right)$. Corollary 2.5 may be extended to $(m, n)$ satisfying $(m-1)(n-1) \leq$ $\min \{m, n\}$. However, the only such pairs are $(1, n),(m, 1),(2,3),(3,2)$. Since $\operatorname{Lat}\left(V, V^{n}\right)$, Lat $\left(V^{m}, V\right)$ are described by Corollary 2.2, Corollary 2.5 may not be extended to other interesting cases.

For $m, n$ arbitrary, we start with subspaces in Lat $\left(V^{m}, V^{n}\right)$ corresponding, in the sense of Lemma 2.3, to subspaces reducing under $V$. The next result describes $\operatorname{Lat}\left(P_{\mathcal{N} \ominus V^{(m-1)(n-1)} \mathcal{N}} V^{m}, P_{\mathcal{N} \ominus V^{(m-1)(n-1)} \mathcal{N}} V^{n}\right)$ in such a case. The following Theorem 2.7 extends the result to the general case. 
Remark 2.6. Let $V=T_{z} \otimes I \in \mathcal{B}\left(H^{2}(\mathbb{T}) \otimes \mathcal{H}\right), m, n$ be relatively prime, positive integers and $\mathcal{H}_{0} \subset \mathcal{H}$. Then $\mathcal{N}=H^{2}(\mathbb{T}) \otimes \mathcal{H}_{0} \in \operatorname{Lat}(V)$. In order to describe all subspaces in $\operatorname{Lat}\left(V^{m}, V^{n}\right)$ corresponding, in the sense of Lemma 2.3 , to $\mathcal{N}$ we need to describe all subspaces $\mathcal{N}_{0}$ satisfying conditions of Theorem 2.4. It is convenient to denote $\mathcal{N}=\sum_{i=0}^{\infty} z^{i} \otimes \mathcal{H}_{0}$. Then $\mathcal{N} \ominus V^{(m-1)(n-1)} \mathcal{N}=\sum_{i=0}^{m n-m-n} z^{i} \otimes \mathcal{H}_{0}$ and

$$
P_{\mathcal{N} \ominus V^{(m-1)(n-1)} \mathcal{N} V^{\alpha}}\left(\sum_{i=0}^{m n-m-n} z^{i} h_{i}\right)=\sum_{i=0}^{m n-m-n-\alpha} z^{i+\alpha} h_{i}
$$

for $\alpha<(m-1)(n-1)$. Let $f(z)=\sum_{i=0}^{m n-m-n} z^{i} h_{i}$ be an arbitrary vector in $\mathcal{N} \ominus V^{(m-1)(n-1)} \mathcal{N}$. The minimal subspace of $\mathcal{N} \ominus V^{(m-1)(n-1)} \mathcal{N}$ invariant under $P_{\mathcal{N} \ominus V^{(m-1)(n-1)} \mathcal{N}} V^{\alpha}$, for $\alpha=m, n$, generated by $f$ is

$$
\mathcal{N}_{f}=\overline{\operatorname{span}}\left\{\sum_{i=0}^{m n-m-n-\beta} z^{i+\beta} h_{i}: \beta=k m+l n<(m-1)(n-1)\right\} .
$$

Note that $\{\beta: \beta=k m+l n<(m-1)(n-1)\}$ is of finite cardinality and so $\operatorname{dim} \mathcal{N}_{f}<\infty$. On the other hand, since $\mathcal{H}$, and so $H^{2}(\mathbb{T}) \otimes \mathcal{H}$ are separable, subspaces of $\mathcal{N} \ominus V^{(m-1)(n-1)} \mathcal{N}$ invariant under $P_{\mathcal{N} \ominus V^{(m-1)(n-1)}} \mathcal{N}^{\alpha}$, for $\alpha=m, n$, are precisely those of the form

$$
\overline{\operatorname{span}}\left\{\mathcal{N}_{f_{i}}:\left\{f_{i}\right\}_{i \geq 0} \subset \mathcal{N} \ominus V^{(m-1)(n-1)} \mathcal{N}\right\} .
$$

Note that if $\operatorname{dim} \mathcal{H}<\infty$, then $\operatorname{dim} \mathcal{N} \ominus V^{(m-1)(n-1)} \mathcal{N}<\infty$.

The following result, Remark 2.6 and Theorem $1.2 \operatorname{describe} \operatorname{Lat}\left(V^{m}, V^{n}\right)$ in the general case.

Theorem 2.7. Let $V=T_{z} \otimes I \in \mathcal{B}\left(H^{2}(\mathbb{T}) \otimes \mathcal{H}\right)$ and $m, n$ be relatively prime, positive integers. The subspaces jointly invariant under $\left(V^{m}, V^{n}\right)$ are precisely those of the form

$$
\hat{\theta}\left(H_{0} \oplus\left(z^{(m-1)(n-1)} H^{2}(\mathbb{T}) \otimes \mathcal{H}_{0}\right)\right)
$$

where $\hat{\theta}$ is an inner function with initial space $H^{2}(\mathbb{T}) \otimes \mathcal{H}_{0}$ and $H_{0}$ is a subspace of $\sum_{i=0}^{m n-m-n} z^{i} \otimes \mathcal{H}_{0}$ invariant under $P_{\sum_{i=0}^{m n-m-n} z^{i} \otimes \mathcal{H}_{0}} V^{\alpha}$ for $\alpha=$ $m, n$.

Proof. By Theorem 1.2 an arbitrary subspace invariant under $V$ is of the form $\mathcal{N}=\hat{\theta}\left(H^{2}(\mathbb{T}) \otimes \mathcal{H}\right)$ where $\hat{\theta}$ is an inner function. Recall that $(\hat{\theta} f)\{z \mapsto$ $\theta(z) f(z)\}$ for $f \in H^{2}(\mathbb{T}) \otimes \mathcal{H}$ where $\theta(z)$ are partial isometries on $\mathcal{H}$ with the same initial space $\mathcal{H}_{0}$ for almost every $z \in \mathbb{T}$. Hence $\{z \mapsto \theta(z) f(z)\}=\{z \mapsto$ $\left.\theta(z) P_{\mathcal{H}_{0}} f(z)\right\}$ where $P_{\mathcal{H}_{0}} \in \mathcal{B}(\mathcal{H})$. In other words, $\hat{\theta} f=\hat{\theta}\left(I \otimes P_{\mathcal{H}_{0}}\right) f$ for any $f \in H^{2}(\mathbb{T}) \otimes \mathcal{H}$. Since $I \otimes P_{\mathcal{H}_{0}}=P_{H^{2}(\mathbb{T}) \otimes \mathcal{H}_{0}}$, we get $\mathcal{N}=\hat{\theta}\left(H^{2}(\mathbb{T}) \otimes \mathcal{H}\right)=$ $\hat{\theta}\left(I \otimes P_{\mathcal{H}_{0}}\right)\left(H^{2}(\mathbb{T}) \otimes \mathcal{H}\right)=\hat{\theta}\left(H^{2}(\mathbb{T}) \otimes \mathcal{H}_{0}\right)$.

Let us denote for convenance $M=(m-1)(n-1)$. Let $f \in \mathcal{N} \ominus V^{M} \mathcal{N}$ and $g \in \mathcal{N}$. Then $f=\left(\hat{\theta} f_{0}\right)\left\{z \mapsto \theta(z) f_{0}(z)\right\}$ and $g=\left(\hat{\theta} g_{0}\right)\left\{z \mapsto \theta(z) g_{0}(z)\right\}$ for some $f_{0}, g_{0} \in H^{2}(\mathbb{T}) \otimes \mathcal{H}_{0}$. Since $\hat{\theta}$ commutes with $V$, we get $V^{M} g:\{z \mapsto$ 
$\left.\theta(z) z^{M} g_{0}(z)\right\}$. Since $f_{0}(z), g_{0}(z) \in \mathcal{H}_{0}$ and $\left.\theta(z)\right|_{\mathcal{H}_{0}}$ is an isometry for almost any $z \in \mathbb{T}$, we get

$$
\begin{aligned}
0=\left\langle f, V^{M} g\right\rangle & =\int_{\mathbb{T}}\left\langle\theta(z) f_{0}(z), \theta(z) z^{M} g_{0}(z)\right\rangle_{\mathcal{H}} d m \\
& =\int_{\mathbb{T}}\left\langle f_{0}(z), z^{M} g_{0}(z)\right\rangle_{\mathcal{H}} d m=\left\langle f_{0}, V^{M} g_{0}\right\rangle .
\end{aligned}
$$

Hence $f_{0} \in\left(H^{2}(\mathbb{T}) \otimes \mathcal{H}_{0}\right) \ominus V^{M}\left(H^{2}(\mathbb{T}) \otimes \mathcal{H}_{0}\right)=\sum_{i=0}^{M-1} z^{i} \otimes \mathcal{H}_{0}$ and so

$$
\mathcal{N} \ominus V^{M} \mathcal{N}=\hat{\theta}\left(\sum_{i=0}^{M-1} z^{i} \otimes \mathcal{H}_{0}\right)
$$

Since $\hat{\theta}$ is an isometry on $H^{2}(\mathbb{T}) \otimes \mathcal{H}_{0}$, the subspace $\mathcal{N} \ominus V^{M} \mathcal{N}$ is orthogonal to $\hat{\theta}\left(\sum_{i=M}^{\infty} z^{i} \otimes \mathcal{H}_{0}\right)$ and

$$
P_{\mathcal{N} \ominus V^{M} \mathcal{N}} \hat{\theta}=P_{\mathcal{N} \ominus V^{M} \mathcal{N}} \hat{\theta} P_{\sum_{i=0}^{M-1} z^{i} \otimes \mathcal{H}_{0}}=\hat{\theta} P_{\sum_{i=0}^{M-1} z^{i} \otimes \mathcal{H}_{0}} .
$$

Note that for any $\mathcal{N}_{0} \subset \mathcal{N} \ominus V^{M} \mathcal{N}$ there is $H_{0} \subset \sum_{i=0}^{M-1} z^{i} \otimes \mathcal{H}_{0}$ such that $\mathcal{N}_{0}=\hat{\theta} H_{0}$. Since $\hat{\theta}$ commutes with $V$ and from (2.1) we get $P_{\mathcal{N} \ominus V^{M} \mathcal{N} V^{\alpha} \hat{\theta} H_{0}}$ $=P_{\mathcal{N} \ominus V^{M} \mathcal{N}} \hat{\theta} V^{\alpha} H_{0}=\hat{\theta} P_{\sum_{i=0}^{M-1} z^{i} \otimes \mathcal{H}_{0}} V^{\alpha} H_{0}$. Therefore $\mathcal{N}_{0}$ is invariant under $P_{\mathcal{N} \ominus V^{M} \mathcal{N} V^{\alpha}}$ if and only if $H_{0}$ is invariant under $P_{\sum_{i=0}^{M-1} z^{i} \otimes \mathcal{H}_{0}} V^{\alpha}$. Theorem 2.4 finishes the proof.

\section{Pairs Satisfying $V_{1}^{m}=U V_{2}^{n}$}

In the section we show that for any pair of commuting isometries there is a minimal extension to a pair of commuting isometries satisfying the equality

$$
V_{1}^{m}=U V_{2}^{n}
$$

where $m, n$ are positive integers and $U$ is a unitary operator commuting with $V_{1}, V_{2}$. One can check that one way that isometric pairs $\left(V_{1}, V_{2}\right)$ satisfying $(\dagger)$ arise is as $\left(V_{1}, V_{2}\right)=\left(U^{k} V^{n}, U^{l} V^{m}\right)$ where $V$ is an isometry and $U$ is a unitary operator commuting with $V$. In the next section we extend pairs satisfying $(\dagger)$ to pairs of the form as above, i.e. $\left(U^{k} V^{m}, U^{l} V^{n}\right)$ where $U$ is a unitary operator commuting with an isometry $V$.

The extension to a pair satisfying $(\dagger)$ does exist since there exists an extension to a pair of unitary operators, which clearly satisfy $(\dagger)$. However, the minimality of the extension is important. Precisely, we are mostly interested in the case when the isometric factor $V$ in the formula of the final extension $\left(U^{k} V^{m}, U^{l} V^{n}\right)$ is a unilateral shift. An example of a pair of unilateral shifts satisfying $(\dagger)$ are generalized powers defined in [3]. In fact pairs satisfying $(\dagger)$ were motivated by generalized powers. Both examples are compatible pairs of isometries (i.e. such that $P_{\operatorname{ran}\left(V_{1}^{m}\right)} P_{\operatorname{ran}\left(V_{2}^{n}\right)}=P_{\operatorname{ran}\left(V_{2}^{n}\right)} P_{\operatorname{ran}\left(V_{1}^{m}\right)}$ for any $m, n>0)$. The model of compatible pairs is known, described in [3,10]. However, not all pairs satisfying $(\dagger)$ are compatible, as in the following example. 
Example 3.1. Let $H=H^{2}(\mathbb{T}) \ominus \mathbb{C}(1-z)$ which is invariant under $T_{z^{i}}$ for $i \geq 2$. Hence we may define $V_{1}=\left.T_{z^{2}}\right|_{H}, V_{2}=\left.T_{z^{3}}\right|_{H}$. Then $V_{1}^{3}=V_{2}^{2}$. One can check that $\operatorname{ker} V_{1}^{*}=\overline{\operatorname{span}}\left\{\frac{\sqrt{2}}{2}(1+z), \frac{\sqrt{2}}{2}\left(z^{2}-z^{3}\right)\right\}$ and $\operatorname{ker} V_{2}^{*}=$ $\overline{\operatorname{span}}\left\{\frac{\sqrt{2}}{2}(1+z), z^{2}, \frac{\sqrt{2}}{2}\left(z^{3}-z^{4}\right)\right\}$. Hence $\operatorname{ran}\left(V_{1}\right)=\overline{\operatorname{span}}\left\{\frac{\sqrt{2}}{2}\left(z^{2}+z^{3}\right), z^{n}: n \geq\right.$ $4\}$ and $\operatorname{ran}\left(V_{2}\right)=\overline{\operatorname{span}}\left\{\frac{\sqrt{2}}{2}\left(z^{3}+z^{4}\right), z^{n}: n \geq 5\right\}$. Thus $P_{\operatorname{ran}\left(V_{1}\right)} P_{\operatorname{ran}\left(V_{2}\right)} z^{4}=$ $P_{\operatorname{ran}\left(V_{1}\right)} \frac{1}{2}\left(z^{3}+z^{4}\right)=\frac{\sqrt{2}}{4}\left(z^{2}+z^{3}\right)+\frac{1}{2} z^{4}$ while $P_{\operatorname{ran}\left(V_{2}\right)} P_{\operatorname{ran}\left(V_{1}\right)} z^{4}=P_{\operatorname{ran}\left(V_{2}\right)} z^{4}=$ $\frac{1}{2}\left(z^{3}+z^{4}\right)$.

Before we construct the extension, let us investigate properties of pairs satisfying $(\dagger)$.

Remark 3.2. Let $\left(V_{1}, V_{2}\right)$ be a pair of commuting isometries. The following conditions are equivalent.

- $\left(V_{1}, V_{2}\right)$ satisfy $(\dagger)$

- $V_{2}^{* n} V_{1}^{m}$ is unitary,

- $\operatorname{ran}\left(V_{1}^{m}\right)=\operatorname{ran}\left(V_{2}^{n}\right)$.

Moreover, a pair satisfying the conditions above admit the Wold-Słociński decomposition. Precisely, it decomposes between a pair of unitary operators and a pair of unilateral shifts.

Note that $U=V_{2}^{* n} V_{1}^{m}$ from $(\dagger)$ and so $V_{2}^{* n} V_{1}^{m}$ is unitary. Conversely, if $V_{2}^{* n} V_{1}^{m}$ is unitary, then, by Remark 1.4 , it commutes with $V_{1}, V_{2}$. Hence the pair satisfies $(\dagger)$ with $U:=V_{2}^{* n} V_{1}^{m}$. We have shown that the pair satisfies $(\dagger)$ if and only if $V_{2}^{* n} V_{1}^{m}$ is unitary. On the other hand, $V_{2}^{* n} V_{1}^{m}$ is an isometry if and only if $\operatorname{ran}\left(V_{1}^{m}\right) \subset \operatorname{ran}\left(V_{2}^{n}\right)$. Thus $V_{2}^{* n} V_{1}^{m}$ is unitary if and only if $\operatorname{ran}\left(V_{1}^{m}\right)=\operatorname{ran}\left(V_{2}^{n}\right)$.

Recall that by Theorem 1.1 a maximal subspace reducing an isometry $V_{i}$ to a unitary operator is $H_{u i}=\bigcap_{i>0} \operatorname{ran}\left(V_{i}^{n}\right)$. Since the sequence $\left\{\operatorname{ran}\left(V_{i}^{n}\right)\right\}_{i>0}$ is decreasing the equality $\operatorname{ran}\left(V_{1}^{m}\right)=\operatorname{ran}\left(V_{2}^{n}\right)$ yields $H_{u 1}=$ $H_{u 2}$. Hence $H_{u 1}=H_{u 2}$ reduces $\left(V_{1}, V_{2}\right)$ to a pair of unitary operators and $H \ominus H_{u 1}=H \ominus H_{u 2}$ reduces $\left(V_{1}, V_{2}\right)$ to a pair of unilateral shifts.

By Remark 3.2 for any extension of $\left(V_{1}, V_{2}\right)$ to a pair $\left(\widehat{V}_{1}, \widehat{V}_{2}\right)$ satisfying $(\dagger)$ the operator $\widehat{V}_{2}^{* n} \widehat{V}_{1}^{m}$ is unitary. However, note that $\widehat{V}_{2}^{* n} \widehat{V}_{1}^{m}$ is not a unitary dilation of $V_{2}^{* n} V_{1}^{m}$. Indeed, by Remark 1.4

$$
\left.P_{H}\left(\widehat{V}_{2}^{* n} \widehat{V}_{1}^{m}\right)^{j}\right|_{H}=\left.P_{H}\left(\widehat{V}_{2}^{* n}\right)^{j}\left(\widehat{V}_{1}^{m}\right)^{j}\right|_{H}=\left(V_{2}^{* n}\right)^{j}\left(V_{1}^{m}\right)^{j} .
$$

Since $\left(V_{2}^{* n}\right)^{j}\left(V_{1}^{m}\right)^{j} \neq\left(V_{2}^{* n} V_{1}^{m}\right)^{j}$ for $j \geq 2$ in general $\widehat{V}_{2}^{* n} \widehat{V}_{1}^{m}$ is not a dilation of $V_{2}^{* n} V_{1}^{m}$. However, if $V_{2}^{* n} V_{1}^{m}$ is an isometry then by Remark 1.4 $\left(V_{2}^{* n}\right)^{j}\left(V_{1}^{m}\right)^{j}=\left(V_{2}^{* n} V_{1}^{m}\right)^{j}$ for $j \in \mathbb{Z}$ and $\widehat{V}_{2}^{* n} \widehat{V}_{1}^{m}$ is a unitary dilation of $V_{2}^{* n} V_{1}^{m}$.

Let us investigate the case when $V_{2}^{* n} V_{1}^{m}$ is an isometry more thoroughly.

Remark 3.3. If a commuting pair of isometries $\left(V_{1}, V_{2}\right)$ on a Hilbert space $H$ is extended to a commuting pair of isometries $\left(\widehat{V}_{1}, \widehat{V}_{2}\right)$ on a Hilbert space $\widehat{H}$ satisfying $\widehat{V}_{1}^{m}=\widehat{U} \widehat{V}_{2}^{n}$ where $\widehat{U} \in \mathcal{B}(\widehat{H})$ is a unitary operator commuting with $\widehat{V}_{1}, \widehat{V}_{2}$ then the following conditions are equivalent: 
1. $V_{2}^{* n} V_{1}^{m}$ is an isometry.

2. $\widehat{U} H \subset H$,

3. $V_{2}^{* n} V_{1}^{m} h=\widehat{U} h$ for any $h \in H$,

4. $\left.\widehat{U}\right|_{H}$ may be considered as an operator on $H$ and $\left.\widehat{U}\right|_{H}$ commutes with $V_{1}, V_{2}$

$(1) \Rightarrow(2)$ Note that $P_{H} \widehat{U} h=P_{H} \widehat{V}_{2}^{* n} \widehat{V}_{1}^{m} h=V_{2}^{* n} V_{1}^{m} h$ for any $h \in H$. Since $\widehat{U}$ and $V_{2}^{* n} V_{1}^{m}$ are isometries, we may reduce the projection and get $\widehat{U} h=V_{2}^{* n} V_{1}^{m} h \in H$.

$(2) \Rightarrow(3)$ Note that $\widehat{V}_{2}^{* n} \widehat{V}_{1}^{m}=\widehat{U}$. If $\widehat{U} H \subset H$ then $V_{2}^{* n} V_{1}^{m} h=$ $P_{H} \widehat{V}_{2}^{* n} \widehat{V}_{1}^{m} h=P_{H} \widehat{U} h=\widehat{U} h$ for any $h \in H$.

$(3) \Rightarrow(1)$ is trivial.

$(2) \Rightarrow(4)$ By the assumption $\left.\widehat{U}\right|_{H}$ indeed may be considered as an operator on $H$. Hence and since $\widehat{V}_{1} h=V_{1} h$ for any $h \in H$ we get $\widehat{V}_{1} \widehat{U} h=$ $V_{1} \widehat{U} h$ for any $h \in H$. Eventually, $\widehat{U} V_{1} h=\widehat{U} \widehat{V}_{1} h=\widehat{V}_{1} \widehat{U} h=V_{1} \widehat{U} h$. The proof for $V_{2}$ is similar.

$(4) \Rightarrow(2)$ is trivial.

Let us show an example where $H$ is not invariant under $\widehat{U}$.

Example 3.4. Let $H=H^{2}(\mathbb{T}) \otimes \mathbb{C}^{2}$ and $V_{1}=T_{z} \otimes I, U=I \otimes \mathcal{U}$ where $\mathcal{U}=\left[\begin{array}{ll}0 & 1 \\ 1 & 0\end{array}\right]$. Set $\mathcal{M}=H \ominus \mathbb{C} f$ where $f=1 \otimes\left[\begin{array}{l}1 \\ 0\end{array}\right]$. Then $U\left(1 \otimes\left[\begin{array}{l}0 \\ 1\end{array}\right]\right)=f$, so $\mathcal{M}$ is not invariant under $U$. However, $\operatorname{ran}\left(V_{1} U\right) \subset \operatorname{ran}\left(V_{1}\right)=\operatorname{ran}\left(T_{z} \otimes I\right)=$ $\sum_{i=1}^{\infty} z^{i} \otimes \mathbb{C}^{2} \subset \mathcal{M}$. Thus $\mathcal{M}$ is invariant under $V_{1}$ and $V_{2}:=V_{1} U=T_{z} \otimes \mathcal{U}$ but it is not invariant under $U$.

Assume that a pair $\left(V_{1}, V_{2}\right)$ and an extension $\left(\widehat{V}_{1}, \widehat{V}_{2}\right)$ satisfying $(\dagger)$ do not meet conditions in Remark 3.3. Since $\widehat{V}_{i}$ commutes with $\widehat{U}$ and $\widehat{V}_{i} H=$ $V_{i} H \subset H$ for $i=1,2$ the space $\tilde{H}:=\overline{\operatorname{span}}\left\{\widehat{U}^{n} H: n \geq 0\right\}$ is invariant under $\widehat{V}_{1}, \widehat{V}_{2}$. Hence, we may define $\tilde{V}_{1}:=\left.\widehat{V}_{1}\right|_{\tilde{H}}, \tilde{V}_{2}:=\left.\widehat{V}_{2}\right|_{\tilde{H}} \in \mathcal{B}(\tilde{H})$. Note that $\left(\widehat{V}_{1}, \widehat{V}_{2}\right)$ is an extension of $\left(\tilde{V}_{1}, \tilde{V}_{2}\right)$ and $\tilde{H}$ is invariant under $\widehat{U}$. Hence, the pair $\left(\tilde{V}_{1}, \tilde{V}_{2}\right)$ and the extension $\left(\widehat{V}_{1}, \widehat{V}_{2}\right)$ satisfy conditions of Remark 3.3. We construct the extension of an arbitrary pair $\left(V_{1}, V_{2}\right)$ to a pair $\left(\widehat{V}_{1}, \widehat{V}_{2}\right)$ satisfying $(\dagger)$ in two steps:

1. An arbitrary pair of commuting isometries $\left(V_{1}, V_{2}\right)$ is extended to a commuting pair $\left(\tilde{V}_{1}, \tilde{V}_{2}\right)$ such that $\tilde{V}_{2}^{* n} \tilde{V}_{1}^{m}$ is an isometry. Then $\tilde{V}_{2}^{* n} \tilde{V}_{1}^{m}$ commutes with $\tilde{V}_{1}, \tilde{V}_{2}$ by Remark 1.4 .

2. Since $\tilde{V}_{2}^{* n} \tilde{V}_{1}^{m}$ is an isometry, its unitary dilation $\widehat{U}$ is in fact an extension. Moreover, $\widehat{U}$ commutes with $\left(\widehat{V}_{1}, \widehat{V}_{2}\right)$-an isometric extensions of $\left(\tilde{V}_{1}, \tilde{V}_{2}\right)$.

Proposition 3.5. For any pair of commuting isometries $\left(V_{1}, V_{2}\right)$ on a Hilbert space $H$ and positive integers $m, n$, there is an extension to a commuting pair of isometries $\left(\widehat{V}_{1}, \widehat{V}_{2}\right)$ on a Hilbert space $\widehat{H}$ satisfying $\widehat{V}_{1}^{m}=\widehat{U} \widehat{V}_{2}^{n}$ where $\widehat{U} \in$ $\mathcal{B}(\widehat{H})$ is a unitary operator commuting with $\widehat{V}_{1}, \widehat{V}_{2}$. Moreover, the extension may be chosen to be minimal. 
Proof. The construction is done in the two steps described before the proposition.

1. Let $\breve{V}_{2} \in \mathcal{B}(\breve{H})$ be the minimal unitary extension of $V_{2}$, and let $\breve{V}_{1}$ be an isometric extension of $V_{1}$ on $\breve{H}$ commuting with $\breve{V}_{2}$. Define $\tilde{H}=$ $\overline{\operatorname{span}}\left\{\breve{U}{ }^{i} H, i \geq 0\right\}$ and $\tilde{U}=\left.\breve{U}\right|_{\tilde{H}}$ where $\breve{U}=\breve{V}_{2}^{* n} \breve{V}_{1}^{m}$. Note that $\breve{U}$ and consequently also $\tilde{U}$ are isometries. Thus, by Remark 1.4, $\breve{U}$ commutes with $\breve{V}_{1}, \breve{V}_{2}$. Hence and since $H \in \operatorname{Lat}\left(\breve{V}_{1}, \breve{V}_{2}\right)$ we get $\tilde{H} \in \operatorname{Lat}\left(\breve{V}_{1}, \breve{V}_{2}\right)$. Subsequently $\tilde{V}_{1}:=\left.\breve{V}_{1}\right|_{\tilde{H}}, \tilde{V}_{2}:=\left.\breve{V}_{2}\right|_{\tilde{H}}$ are commuting isometries on $\tilde{H}$ commuting also with $\tilde{U} \in \mathcal{B}(\tilde{H})$. Moreover, $\tilde{V}_{1}^{m}=\tilde{U} \tilde{V}_{2}^{n}$ and hence $\tilde{V}_{2}^{* n} \tilde{V}_{1}^{m}=\tilde{U}$ is an isometry.

2. Let $\widehat{U} \in \mathcal{B}(\widehat{H})$ be the minimal unitary extension of $\tilde{U}$ and $\left(\widehat{V}_{1}, \widehat{V}_{2}\right)$ be a commuting isometric extension of $\left(\tilde{V}_{1}, \tilde{V}_{2}\right)$ on $\widehat{H}$, commuting also with $\widehat{U}$ - see [13, Proposition 6.3, chap.1] for details of such a construction. Note that $\left.\widehat{V}_{1}^{m} \widehat{U}^{i}\right|_{\tilde{H}}=\left.\widehat{U}^{i} \widehat{V}_{1}^{m}\right|_{\tilde{H}}=\widehat{U}^{i} \tilde{V}_{1}^{m}=\widehat{U}^{i} \tilde{U} \tilde{V}_{2}^{n}=\left.\widehat{U}^{i+1} \widehat{V}_{2}^{n}\right|_{\tilde{H}}=$ $\left.\widehat{U} \widehat{V}_{2}^{n} \widehat{U}^{i}\right|_{\tilde{H}}$, and so $\left.\widehat{V}_{1}^{m}\right|_{\widehat{U}^{i} \tilde{H}}=\left.\widehat{U}_{V_{2}^{n}}\right|_{\widehat{U}^{i} \tilde{H}}$ for any $i \in \mathbb{Z}$. On the other hand, by the minimality $\widehat{H}=\overline{\operatorname{span}}\left\{\widehat{U} \widehat{U}^{i} \tilde{H}: i \leq 0\right\}$. Hence $\widehat{V}_{1}^{m}=\widehat{U} \widehat{V}_{2}^{n}$.

For the minimality, let $\widehat{\mathcal{H}} \subset \widehat{H}$ be such that $\left(\widehat{\mathcal{V}}_{1}=\left.\widehat{V}_{1}\right|_{\widehat{\mathcal{H}}}, \widehat{\mathcal{V}}_{2}=\left.\widehat{V}_{2}\right|_{\widehat{\mathcal{H}}}\right)$ on $\widehat{\mathcal{H}}$ is an extensions of $\left(V_{1}, V_{2}\right)$ satisfying the statement. Note that $\widehat{\mathcal{U}}:=$ $\widehat{\mathcal{V}}_{2}^{* n} \widehat{\mathcal{V}}_{1}^{m}=P_{\widehat{\mathcal{H}}} \widehat{V}_{2}^{* n} \widehat{V}_{1}^{m}=\left.P_{\widehat{\mathcal{H}}} \widehat{U}\right|_{\widehat{\mathcal{H}}}$. However, by the assumption $\widehat{\mathcal{U}}$ as well as $\widehat{U}$ are unitary operators and so we may reduce the projection. Hence $\widehat{\mathcal{U}}=\left.\widehat{U}\right|_{\widehat{\mathcal{H}}}$ and in particular $\left.\widehat{\mathcal{U}}\right|_{H}=\left.\widehat{U}\right|_{H}=\left.\breve{U}\right|_{H}$. Thus $\tilde{H}=\overline{\operatorname{span}}\left\{\widehat{\mathcal{U}}^{i} H, i \geq 0\right\} \subset \widehat{\mathcal{H}}$ and $\tilde{U}=\left.\widehat{\mathcal{U}}\right|_{\tilde{H}}$. Consequently $\widehat{\mathcal{U}}=\left.\widehat{U}\right|_{\widehat{\mathcal{H}}}$ is a unitary extension of $\tilde{U}$. Since $\widehat{\mathcal{H}} \subset \widehat{H}$ by the minimality of $\widehat{U}$ we get $\widehat{\mathcal{H}}=\widehat{H}$.

Let us show an example of a pair of unilateral shifts such that the extension constructed in Proposition 3.5, so a minimal extension, is a pair of unitary operators.

Example 3.6. Let $\left(L_{w}, L_{z}\right)$ denote operators of multiplication by independent variables $w, z$ on $L^{2}\left(\mathbb{T}^{2}\right)$ and $\mathcal{M}:=\left\{f \in L^{2}\left(\mathbb{T}^{2}\right): \hat{f}_{i, j}=0\right.$ for $\left.(i, j) \in \mathbb{Z}^{2} \backslash Z\right\}$ where $Z:=\left\{(i, j) \in \mathbb{Z}^{2}: j \geq 0\right.$ and $\left.i \geq-(0+\cdots+j)\right\}$ as in the picture.

Then $\mathcal{M} \in \operatorname{Lat}\left(L_{w}, L_{z}\right)$ and $V_{1}=\left.L_{w}\right|_{\mathcal{M}}, V_{2}=\left.L_{z}\right|_{\mathcal{M}} \in \mathcal{B}(\mathcal{M})$ are unilateral shifts. Moreover, $V_{2}^{* n} V_{1}^{m}=\left.P_{\mathcal{M}} L_{\bar{z}}^{n} L_{w}^{m}\right|_{\mathcal{M}}$ for any positive integers $m, n$. Note that a minimal isometric extension of $\left.P_{\mathcal{M}} L_{\bar{z}}^{n} L_{w}^{m}\right|_{\mathcal{M}}$ is the restriction $\left.L_{\bar{z}}^{n} L_{w}^{m}\right|_{\tilde{\mathcal{M}}}$ where $\tilde{\mathcal{M}}=\overline{\operatorname{span}}\left\{\left(L_{\bar{z}}^{n} L_{w}^{m}\right)^{s} \mathcal{M}: s \geq 0\right\}$. By Remark 1.4, $\left(L_{\bar{z}}^{n} L_{w}^{m}\right)^{s}=$ 
$L_{\bar{z}}^{s n} L_{w}^{s m}$ for $s \geq 1$. Hence $\left(\left.L_{\bar{z}}^{n} L_{w}^{m}\right|_{\tilde{\mathcal{M}}}\right)^{s}=\left.\left(L_{\bar{z}}^{n} L_{w}^{m}\right)^{s}\right|_{\tilde{\mathcal{M}}}=\left.L_{\bar{z}}^{s n} L_{w}^{s m}\right|_{\tilde{\mathcal{M}}}$. Let us show that $\tilde{\mathcal{M}}=L^{2}\left(\mathbb{T}^{2}\right)$. Consider $w^{k} z^{l}$ for an arbitrary $(k, l) \in \mathbb{Z}^{2}$. Note that there are $(i, j) \in Z$ and a positive integer $s$ such that $(k, l)=(i+s m, j-s n)$. Hence $w^{i} z^{j} \in \mathcal{M}$ and $\left(L_{\bar{z}}^{n} L_{w}^{m}\right)^{s} w^{i} z^{j}=L_{\bar{z}}^{s n} L_{w}^{s m} w^{i} z^{j}=w^{i+s m} z^{j-s n}=w^{k} z^{l}$. Thus indeed $\tilde{\mathcal{M}}=L^{2}\left(\mathbb{T}^{2}\right)$, and so a minimal extension of $\left(V_{1}, V_{2}\right)$ to a pair of commuting isometries satisfying $(\dagger)$ is a pair of unitary operators $\left(L_{w}, L_{z}\right)$.

Operators of multiplication by independent variables on the Hardy space over the torus have an extension satisfying $(\dagger)$ which is a pair of unilateral shifts.

Example 3.7. Let $\left(T_{w}, T_{z}\right)$ denote operators of multiplication by independent variables $w, z$ on the Hardy space over the torus $H^{2}\left(\mathbb{T}^{2}\right)$. The minimal extension of $\left(T_{w}, T_{z}\right)$ to a commuting pair of isometries $\left(\widehat{T}_{w}, \widehat{T}_{z}\right)$ satisfying $\widehat{T}_{w}=\widehat{U} \widehat{T}_{z}$ is $\widehat{T}_{w}=\left.L_{w}\right|_{\Delta^{2}\left(\mathbb{T}^{2}\right)}, \widehat{T}_{z}=\left.L_{z}\right|_{\Delta^{2}\left(\mathbb{T}^{2}\right)}$ where

$$
\Delta^{2}\left(\mathbb{T}^{2}\right):=\left\{f \in L^{2}\left(\mathbb{T}^{2}\right): \hat{f}_{i, j}=0 \text { for } j<-i\right\}
$$

and $\widehat{U}=\left.L_{\bar{z} w}\right|_{\Delta^{2}\left(\mathbb{T}^{2}\right)}$.

To get the extension of $\left(T_{w}, T_{z}\right)$ to a pair satisfying $(\dagger)$ for arbitrary $m, n$ redefine $\Delta^{2}\left(\mathbb{T}^{2}\right):=\left\{f \in L^{2}\left(\mathbb{T}^{2}\right): \hat{f}_{i, j}=0\right.$ for $\left.m j<-n i\right\}$ in Example 3.7.

\section{Extension to a Generalization of Powers of an Isometry}

In this section we construct a minimal extension of an arbitrary pair of commuting isometries $\left(V_{1}, V_{2}\right)$ to a pair of the form

$$
\left(U^{k} V^{n}, U^{l} V^{m}\right)
$$

where $U$ is a unitary operator commuting with an isometry $V$ and integers, positive $m, n, k$ and nonnegative $l$, satisfy $k m-l n=1$. We also describe a model for such pairs. Note that for a pair of positive integers $(m, n)$ there are integers $(k, l)$ such that $k m-l n=1$ if and only if $m, n$ are relatively prime. Moreover, by Remark 1.5 such $(k, l)$ satisfying $0<k<n, 0 \leq l<m$ are unique, hence determined by $(m, n)$.

Consider a pair of commuting isometries $\left(V_{1}, V_{2}\right)$ satisfying $(\dagger)$ for the unitary operator $U$ commuting with $V_{1}, V_{2}$ and relatively prime, positive integers $m, n$. By Fuglede-Putnam theorem $U^{*}$ commutes with $V_{1}, V_{2}$. Hence the condition $(\dagger)$ which by $k m-l n=1$ may be stated as $V_{1}^{m}=U^{k m-l n} V_{2}^{n}$ is equivalent to

$$
\left(U^{* k} V_{1}\right)^{m}=\left(U^{* l} V_{2}\right)^{n} .
$$

Note that conditions $\left(\dagger^{\prime}\right)$ and $\left(\dagger^{\prime}\right)$ are equivalent but $\left(\dagger^{\prime}\right)$ requires $m, n$ to be relatively prime. The pair $\left(U^{k} V^{n}, U^{l} V^{m}\right)$ satisfies ( $\left.\dagger^{\prime}\right)$ for any isometry $V$ and a unitary operator $U$ provided they commute. Indeed, as assumed for pairs satisfying (†), $U$ commutes with $V_{1}=U^{k} V^{n}, V_{2}=U^{l} V^{m}$ and hence it commutes with $V^{m}, V^{n}$. However, by Fuglede-Putnam theorem it doubly commutes with $V^{m}, V^{n}$ and so with $V=V^{* l n} V^{k m}$. Obviously $\left(U^{k} V^{n}, U^{l} V^{m}\right)$ are unilateral shifts if and only if $V$ is a unilateral shift. 
Before we construct the extension, let us investigate properties of pairs of the form $(\ddagger)$ and give their model.

Remark 4.1. Let $\left(V_{1}, V_{2}\right)$ be a pair of commuting isometries and $m, n$ be relatively prime, positive integers with the corresponding integers $0<k<$ $n, 0 \leq l<m$ such that $k m-l n=1$. The pair $\left(V_{1}, V_{2}\right)$ is of the form $(\ddagger)$ if and only if $V_{2}^{* n} V_{1}^{m}$ is a unitary operator and $V_{1}^{* l} V_{2}^{k}$ is an isometry. Then $U=V_{2}^{* n} V_{1}^{m}$ and $V=V_{1}^{* l} V_{2}^{k}$.

Indeed, let $V_{1}=U^{k} V^{n}, V_{2}=U^{l} V^{m}$ for some unitary operator $U$ commuting with an isometry $V$. Then $V^{n}=U^{* k} V_{1}, V^{m}=U^{* l} V_{2}$ and $U$ doubly commutes with $V_{1}, V_{2}$. Hence

$$
\begin{aligned}
V=V^{k m-l n}=\left(V^{n}\right)^{* l}\left(V^{m}\right)^{k} & =\left(U^{* k} V_{1}\right)^{* l}\left(U^{* l} V_{2}\right)^{k} \\
& =V_{1}^{* l} U^{k l} U^{* l k} V_{2}^{k}=V_{1}^{* l} V_{2}^{k}
\end{aligned}
$$

and

$$
\begin{aligned}
V_{2}^{* n} V_{1}^{m}=V^{* m n} U^{* l n} U^{k m} V^{n m} & =V^{* m n} U^{k m-l n} V^{n m} \\
& =V^{* m n} V^{n m} U^{k m-l n}=U .
\end{aligned}
$$

Conversely, assume that $V_{2}^{* n} V_{1}^{m}$ is a unitary operator and $V_{1}^{* l} V_{2}^{k}$ is an isometry. Any commuting pair of isometries satisfies $V_{1}^{m}=U V_{2}^{n}$ for $U:=$ $V_{2}^{* n} V_{1}^{m}$. Since $U$ is unitary, it doubly commutes with $V_{1}, V_{2}$ by Remark 1.4 and Fuglede-Putnam theorem. Hence $U$ commutes with $V:=V_{1}^{* l} V_{2}^{k}$. By Remark $1.4 \mathrm{~V}$ commutes with $V_{1}, V_{2}$ and $V^{m}=V_{1}^{* m l} V_{2}^{m k}$. Further, $m k=$ $n l+1$ yields

$$
V_{1}^{* m l} V_{2}^{m k}=V_{1}^{* m l} V_{2}^{n l+1}=V_{1}^{* m l}\left(V_{2}^{n}\right)^{l} V_{2}=V_{1}^{* m l}\left(U^{*} V_{1}^{m}\right)^{l} V_{2}=U^{* l} V_{2} .
$$

Hence $V_{2}=U^{l} V^{m}$. The proof of $V_{1}=U^{k} V^{n}$ is similar.

We next describe the model for a pair of the form $(\ddagger)$. By Remark 3.2 any pair of commuting isometries satisfying $(\dagger)$ (in particular any pair of the form $(\ddagger)$ ) decomposes between a pair of unitary operators and a pair of unilateral shifts. By Remark 4.1 any pair of commuting unitary operators may be represented in the form $(\ddagger)$ for any relatively prime, positive integers $m, n$. On the other hand, if a pair of commuting unilateral shifts $\left(V_{1}, V_{2}\right)$ equals to $\left(U^{k} V^{n}, U^{l} V^{m}\right)$ for a unitary operator $U$ commuting with an isometry $V$ then $V$ is a unilateral shift. Hence we may assume $V=T_{z} \otimes I \in \mathcal{B}\left(H^{2}(\mathbb{T}) \otimes \mathcal{H}\right)$ and by Remark $1.3 U=I \otimes \mathcal{U}$ where $\mathcal{U} \in \mathcal{B}(\mathcal{H})$ is a unitary operator. Eventually, $V_{1}=T_{z}^{n} \otimes \mathcal{U}^{k}, V_{2}=T_{z}^{m} \otimes \mathcal{U}^{l}$. Summing up we get the model:

Remark 4.2. Pairs of the form $(\ddagger)$ are precisely:

$$
V_{1}=U_{1} \oplus\left(T_{z}^{n} \otimes \mathcal{U}^{k}\right), V_{2}=U_{2} \oplus\left(T_{z}^{m} \otimes \mathcal{U}^{l}\right) \in \mathcal{B}\left(H_{u} \oplus\left(H^{2}(\mathbb{T}) \otimes \mathcal{H}\right)\right)
$$

where $U_{1}, U_{2} \in \mathcal{B}\left(H_{u}\right), \mathcal{U} \in \mathcal{B}(\mathcal{H})$ are unitary operators, $m, n$ are relatively prime, positive integers and $0<k<n, 0 \leq l<m$ are such that $k m-l n=1$. Precisely, $V_{1}=U^{k} V^{n}, V_{2}=U^{l} V^{m}$ where $\bar{U}=U_{2}^{* n} U_{1}^{m} \oplus(I \otimes \mathcal{U}), V=U_{1}^{* l} U_{2}^{k} \oplus$ $\left(T_{z} \otimes I\right)$. Thus invariants of pairs of the form $(\ddagger)$ for a fixed pair of relatively prime, positive integers $m, n$ are Hilbert spaces $H_{u}, \mathcal{H}$ and unitary operators $U_{1}, U_{2} \in \mathcal{B}\left(H_{u}\right), \mathcal{U} \in \mathcal{B}(\mathcal{H})$ where $U_{1}, U_{2}$ commute. 
Let us remark that the model above is not the special case of BergerCoburn-Lebow model in [1]. To compare the models we assume that a pair of commuting isometries $\left(V_{1}, V_{2}\right)$ is of the form $(\ddagger)$ and is completely non unitary (which is assumed in Berger-Coburn-Lebow model). Then it is a pair of unilateral shifts and both models are defined on $H^{2}(\mathbb{T}) \otimes \mathcal{H}$. However, $T_{z} \otimes I=V_{1} V_{2}$ in Berger-Coburn-Lebow model which is not true in the model above.

The main aim of the section is to construct a minimal extension of an arbitrary pair of commuting isometries to a pair of the form $(\ddagger)$. According to the previous section we may reduce the problem to the case where the initial pair satisfy $(\dagger)$. An example of a pair satisfying $(\dagger)$ which is not of the form $(\ddagger)$ is $\left(\left.V_{1}\right|_{\mathcal{M}_{0}},\left.V_{2}\right|_{\mathcal{M}_{0}}\right)$ in Example 2.1. Indeed, it satisfies $(\dagger)$ for $U=I$ but is not a pair of powers of the same isometry since $V$ defined in the example acts on a superspace of $\mathcal{M}_{0}$.

By Remark 4.1 we need to construct an extension of an arbitrary pair of commuting isometries to a pair $\left(\tilde{V}_{1}, \tilde{V}_{2}\right)$ such that $\tilde{V}_{2}^{* \beta} \tilde{V}_{1}^{\alpha}$ are isometric/unitary for the appropriate pairs of powers $(\alpha, \beta)$. In the further part, in the proof of the main theorem of the section we show that for a commuting pair of isometries $\left(V_{1}, V_{2}\right)$ satisfying $(\dagger)$ operators $V_{2}^{* b} V_{1}^{a}$ are isometries for sufficiently large $(a, b)$. On the other hand, $(\alpha, \beta)$ required in Remark 4.1 may not be large enough. Therefore we need the following proposition. Note that if $V_{2}^{* \beta} V_{1}^{\alpha}$ is an isometry, then, by Remark $1.4,\left(V_{2}^{* \beta} V_{1}^{\alpha}\right)^{2}=V_{2}^{* 2 \beta} V_{1}^{2 \alpha}$ and so $V_{2}^{* 2 \beta} V_{1}^{2 \alpha}$ is an isometry, but the converse implication may not hold. For example, if $m=3, n=2$ in Example 2.1, then $\left(\left.V_{2}\right|_{\mathcal{M}_{0}}\right)^{* 2}\left(\left.V_{1}\right|_{\mathcal{M}_{0}}\right)^{2}=\left.V^{2}\right|_{\mathcal{M}_{0}}$, so it is an isometry, but $\left(\left.V_{2}\right|_{\mathcal{M}_{0}}\right)^{*}\left(\left.V_{1}\right|_{\mathcal{M}_{0}}\right)$ vanishes on $\mathcal{M} \ominus V \mathcal{M}$.

Proposition 4.3. Let $\left(V_{1}, V_{2}\right)$ be a commuting pair of isometries on a Hilbert space $H$ such that $V_{2}^{* 2 \beta} V_{1}^{2 \alpha}$ is an isometry. There is a minimal extension $\left(\widetilde{V}_{1}, \widetilde{V}_{2}\right)$ on a Hilbert space $\widetilde{H}$ such that $\widetilde{V}_{2}^{* \beta} \widetilde{V}_{1}^{\alpha}$ is an isometry.

Moreover, the extension preserves the following properties:

- if $V_{i}$ is a unilateral shift, then $\widetilde{V}_{i}$ is a unilateral shift for $i=1,2$,

- if $V_{2}^{* \beta} V_{1}^{\alpha}$ is a co-isometry, then $\widetilde{V}_{2}^{* \beta} \widetilde{V}_{1}^{\alpha}$ is a unitary operator,

- if $V_{2}^{* n} V_{1}^{m}$ is unitary, then $\widetilde{V}_{2}^{* n} \widetilde{V}_{1}^{m}$ is unitary for $m, n \in \mathbb{Z}_{+}$.

Proof. Let

$$
\widetilde{H}:=\{(h, f): h \in H, f \in \mathcal{D}\}
$$

be a cartesian product Hilbert space with $\left\langle\left(h_{1}, f_{1}\right),\left(h_{2}, f_{2}\right)\right\rangle_{\widetilde{H}}=\left\langle h_{1}, h_{2}\right\rangle+$ $\left\langle f_{1}, f_{2}\right\rangle$, and so $\|(h, f)\|_{\widetilde{H}}^{2}=\|h\|^{2}+\|f\|^{2}$ where

$$
\mathcal{D}=\overline{\left(I-V_{2}^{\beta} V_{2}^{* \beta}\right) V_{1}^{\alpha} H}
$$

and $\langle\cdot, \cdot\rangle,\|\cdot\|$ denote the scalar product, the norm on $H$, respectively. Note that $H \subset \widetilde{H}$ in the sense of the equivalence $h \simeq(h, 0)$. Define

$$
\widetilde{W}(h, f)=\left(W h+V_{2}^{* \beta} W f,\left(I-V_{2}^{\beta} V_{2}^{* \beta}\right) W f\right)
$$

for any isometry $W$ commuting with $V_{1}, V_{2}$. In particular (4.1) defines $\left(\widetilde{V}_{1}, \widetilde{V}_{2}\right)$ which we show to satisfy the statement. 
For an arbitrary $f=\lim _{n \rightarrow \infty}\left(I-V_{2}^{\beta} V_{2}^{* \beta}\right) V_{1}^{\alpha} g_{n} \in \mathcal{D}$ by the second claim in Remark 1.4 we get

$$
\begin{aligned}
\left(I-V_{2}^{\beta} V_{2}^{* \beta}\right) W f & =\lim _{n \rightarrow \infty}\left(I-V_{2}^{\beta} V_{2}^{* \beta}\right) W\left(I-V_{2}^{\beta} V_{2}^{* \beta}\right) V_{1}^{\alpha} g_{n} \\
& =\lim _{n \rightarrow \infty}\left(I-V_{2}^{\beta} V_{2}^{* \beta}\right) V_{1}^{\alpha} W g_{n} \in \mathcal{D} .
\end{aligned}
$$

Hence $\widetilde{W}$ is a well defined operator.

Note that if $f \in \mathcal{D} \subset \operatorname{ker} V_{2}^{* \beta}$ then $V_{2}^{* \beta} W f \in \operatorname{ker} W^{*}$ and so

$$
\begin{aligned}
\|\widetilde{W}(h, f)\|_{\widetilde{H}}^{2} & =\|W h\|^{2}+\left\|V_{2}^{* \beta} W f\right\|^{2}+\left\|\left(I-V_{2}^{\beta} V_{2}^{* \beta}\right) W f\right\|^{2} \\
& =\|W h\|^{2}+\left\|V_{2} V_{2}^{* \beta} W f\right\|^{2}+\left\|\left(I-V_{2}^{\beta} V_{2}^{* \beta}\right) W f\right\|^{2} \\
& =\|h\|^{2}+\|W f\|^{2}=\|(h, f)\|_{\widetilde{H}}^{2} .
\end{aligned}
$$

Consequently $\widetilde{W}$ is an isometry.

One can check that for any pair of isometries $W, V$ commuting with $V_{1}, V_{2}$ and between each other

$$
\widetilde{W} \widetilde{V}(h, f)=\left(W V h+V_{2}^{* \beta} W V f,\left(I-V_{2}^{\beta} V_{2}^{* \beta}\right) W V f\right)=\widetilde{W V}(h, f)
$$

where the first coordinate in (4.2) follows by a direct calculation, while the second is a consequence of the second claim in Remark 1.4. Hence $\widetilde{W}, \widetilde{V}$ inherit commutativity. In particular $\widetilde{V}_{1}$ commutes with $\widetilde{V}_{2}$.

Summing up, $\left(\widetilde{V}_{1}, \widetilde{V}_{2}\right)$ is a commuting pair of isometries extending $\left(V_{1}, V_{2}\right)$.

Next we show that $\operatorname{ran}\left(\widetilde{V}_{1}^{\alpha}\right)$ is orthogonal to $\operatorname{ker} \widetilde{V}_{2}^{* \beta}$ which implies that $\widetilde{V}_{2}^{* \beta} \widetilde{V}_{1}^{\alpha}$ is an isometry.

From (4.2) we get $\widetilde{V_{i}} \widetilde{V_{i}^{j}}=\widetilde{V_{i}^{j+1}}$ and hence inductively $\widetilde{V}_{i}^{j}=\widetilde{V_{i}^{j}}$ for $j \geq 0$ and $i=1,2$. Precisely,

$$
\widetilde{V}_{i}^{j}(h, f)=\left(V_{i}^{j} h+V_{2}^{* \beta} V_{i}^{j} f,\left(I-V_{2}^{\beta} V_{2}^{* \beta}\right) V_{i}^{j} f\right) .
$$

Assume $j \geq \alpha$. Since $V_{2}^{* 2 \beta} V_{1}^{2 \alpha}$ is an isometry $\operatorname{ran}\left(V_{1}^{j+\alpha}\right) \subset \operatorname{ran}\left(V_{1}^{2 \alpha}\right) \subset$ $\operatorname{ran}\left(V_{2}^{2 \beta}\right) \subset \operatorname{ran}\left(V_{2}^{\beta}\right)$. Hence and by the second claim in Remark 1.4

$$
\left(I-V_{2}^{\beta} V_{2}^{* \beta}\right) V_{1}^{j}\left(I-V_{2}^{\beta} V_{2}^{* \beta}\right) V_{1}^{\alpha} g=\left(I-V_{2}^{\beta} V_{2}^{* \beta}\right) V_{1}^{j+\alpha} g=0
$$

for any $g \in H$. Since $\left(I-V_{2}^{\beta} V_{2}^{* \beta}\right) V_{1}^{\alpha} g$ represents an arbitrary vector in a dense subset of $\mathcal{D}$ by continuity $\left.\left(I-V_{2}^{\beta} V_{2}^{* \beta}\right) V_{1}^{j}\right|_{\mathcal{D}}=0$. Hence and from (4.3) we get

$$
\begin{aligned}
& \widetilde{V}_{2}^{j}(h, f)=\left(V_{2}^{j} h+V_{2}^{j-\beta} f, 0\right) \text { for } j \geq \beta, \\
& \widetilde{V}_{1}^{j}(h, f)=\left(V_{1}^{j} h+V_{2}^{* \beta} V_{1}^{j} f, 0\right) \text { for } j \geq \alpha,
\end{aligned}
$$

and subsequently

$$
\begin{aligned}
& \operatorname{ran}\left(\widetilde{V}_{1}^{\alpha}\right)=\left\{(h, 0): h \in \operatorname{ran}\left(V_{1}^{\alpha}\right) \oplus V_{2}^{* \beta} V_{1}^{\alpha} \mathcal{D}\right\}, \\
& \operatorname{ran}\left(\widetilde{V}_{2}^{\beta}\right)=\left\{(h, 0): h \in \operatorname{ran}\left(V_{2}^{\beta}\right) \oplus \mathcal{D}\right\}, \\
& \operatorname{ker} \widetilde{V}_{2}^{* \beta}=\left\{(h, f): h \in \operatorname{ker} V_{2}^{* \beta} \ominus \mathcal{D}, f \in \mathcal{D}\right\} .
\end{aligned}
$$


Note that $V_{2}^{* \beta} V_{1}^{\alpha} \mathcal{D}$ is closed since $\left.\left(I-V_{2}^{\beta} V_{2}^{* \beta}\right) V_{1}^{j}\right|_{\mathcal{D}}=0$ yields that $\left.V_{2}^{* \beta} V_{1}^{\alpha}\right|_{\mathcal{D}}$ is an isometry.

We investigate $\mathcal{D}$ to get a more convenient description of $\operatorname{ker} \widetilde{V}_{2}^{* \beta}$. Since $\left(I-V_{2}^{\beta} V_{2}^{* \beta}\right) V_{1}^{\alpha} V_{2}^{\beta}=0$ we get $\mathcal{D}=\overline{\left(I-V_{2}^{\beta} V_{2}^{* \beta}\right) V_{1}^{\alpha} \operatorname{ker} V_{2}^{* \beta}}$. Moreover, for $h \in \operatorname{ker} V_{2}^{* \beta} \ominus V_{1}^{* \alpha} \operatorname{ker} V_{2}^{* \beta}$ and $f \in \operatorname{ker} V_{2}^{* \beta}$ we get $0=\left\langle h, V_{1}^{* \alpha} f\right\rangle=\left\langle V_{1}^{\alpha} h, f\right\rangle$. Thus $V_{1}^{\alpha}\left(\operatorname{ker} V_{2}^{* \beta} \ominus V_{1}^{* \alpha} \operatorname{ker} V_{2}^{* \beta}\right) \subset \operatorname{ran} V_{2}^{\beta}$ and we get further simplification

$$
\mathcal{D}=\overline{\left(I-V_{2}^{\beta} V_{2}^{* \beta}\right) V_{1}^{\alpha} V_{1}^{* \alpha} \operatorname{ker} V_{2}^{* \beta}}=\overline{P_{\operatorname{ker} V_{2}^{* \beta}} P_{\operatorname{ran} V_{1}^{\alpha}} \operatorname{ker} V_{2}^{* \beta}} .
$$

On the other hand,

$$
\left\langle v,\left(I-V_{2}^{\beta} V_{2}^{* \beta}\right) V_{1}^{\alpha} V_{1}^{* \alpha} w\right\rangle=\left\langle v, V_{1}^{\alpha} V_{1}^{* \alpha} w\right\rangle=\left\langle V_{1}^{* \alpha} v, V_{1}^{* \alpha} w\right\rangle
$$

for $v, w \in \operatorname{ker} V_{2}^{* \beta}$. For $w=v \in \operatorname{ker} V_{2}^{* \beta} \ominus \mathcal{D}$ the left hand side of $(\diamond)$ equals 0 and the right hand side is equal to $\left\|V_{1}^{* \alpha} v\right\|^{2}$. Hence $\left\|V_{1}^{* \alpha} v\right\|^{2}=0$ and so $\operatorname{ker} V_{2}^{* \beta} \ominus \mathcal{D} \subset \operatorname{ker} V_{1}^{* \alpha} \cap \operatorname{ker} V_{2}^{* \beta}$. On the other hand, for $v \in \operatorname{ker} V_{1}^{* \alpha} \cap \operatorname{ker} V_{2}^{* \beta}$ and an arbitrary $w \in \operatorname{ker} V_{2}^{* \beta}$ the right hand side of $(\diamond)$ equals 0 which implies that $\operatorname{ker} V_{1}^{* \alpha} \cap \operatorname{ker} V_{2}^{* \beta}$ is orthogonal to a dense subset of $\mathcal{D}$, so the converse inclusion $\operatorname{ker} V_{1}^{* \alpha} \cap \operatorname{ker} V_{2}^{* \beta} \subset \operatorname{ker} V_{2}^{* \beta} \ominus \mathcal{D}$. Thus $\operatorname{ker} V_{2}^{* \beta} \ominus \mathcal{D}=$ $\operatorname{ker} V_{1}^{* \alpha} \cap \operatorname{ker} V_{2}^{* \beta}$ and

$$
\operatorname{ker} \widetilde{V}_{2}^{* \beta}=\left\{(h, f): h \in \operatorname{ker} V_{1}^{* \alpha} \cap \operatorname{ker} V_{2}^{* \beta}, f \in \mathcal{D}\right\} .
$$

Recall that our aim is to show that $\operatorname{ran}\left(\widetilde{V}_{1}^{\alpha}\right)$ is orthogonal to $\operatorname{ker} \widetilde{V}_{2}^{* \beta}$ which by the above and (4.5) is equivalent to $V_{2}^{* \beta} V_{1}^{\alpha} \mathcal{D} \subset H \ominus \operatorname{ker} V_{1}^{* \alpha} \cap$ $\operatorname{ker} V_{2}^{* \beta}=\overline{\operatorname{span}}\left\{\operatorname{ran}\left(V_{1}^{\alpha}\right), \operatorname{ran}\left(V_{2}^{\beta}\right)\right\}$. By the definition of $\mathcal{D}$ it is enough to prove that $V_{2}^{* \beta} V_{1}^{\alpha}\left(I-V_{2}^{\beta} V_{2}^{* \beta}\right) V_{1}^{\alpha} g=V_{2}^{* \beta} V_{1}^{2 \alpha} g-V_{1}^{\alpha} V_{2}^{* \beta} V_{1}^{\alpha} g \in$ $\overline{\operatorname{span}}\left\{\operatorname{ran}\left(V_{1}^{\alpha}\right), \operatorname{ran}\left(V_{2}^{\beta}\right)\right\}$ for any $g \in H$. Since $V_{2}^{* 2 \beta} V_{1}^{2 \alpha}$ is an isometry, by Remark 1.4 it commutes with $V_{2}$ and consequently $V_{2}^{* \beta} V_{1}^{2 \alpha} g=V_{2}^{* 2 \beta} V_{1}^{2 \alpha} V_{2}^{\beta} g=$ $V_{2}^{\beta} V_{2}^{* 2 \beta} V_{1}^{2 \alpha} g \in \operatorname{ran}\left(V_{2}^{\beta}\right)$. The second summand $V_{1}^{\alpha} V_{2}^{* \beta} V_{1}^{\alpha} g$ is trivially in $\operatorname{ran}\left(V_{1}^{\alpha}\right)$.

We showed that $\widetilde{V}_{2}^{* \beta} \widetilde{V}_{1}^{\alpha}$ is an isometry. Let us check the minimality. Assume that $\left(\left.\widetilde{V}_{1}\right|_{\tilde{H}},\left.\widetilde{V}_{2}\right|_{\tilde{H}}\right)$ satisfy the statement where $H \subset \tilde{H} \subset \tilde{H}$ and $\tilde{H} \in \operatorname{Lat}\left(\widetilde{V}_{1}, \widetilde{V}_{2}\right)$. Note that $\left(\left.\widetilde{V}_{2}\right|_{\tilde{H}}\right)^{* \beta}\left(\left.\widetilde{V}_{1}\right|_{\tilde{H}}\right)^{\alpha}=\left.P_{\tilde{H}} \widetilde{V}_{2}^{* \beta} \widetilde{V}_{1}^{\alpha}\right|_{\tilde{H}}$. Since $\left(\left.\widetilde{V}_{2}\right|_{\tilde{H}}\right)^{* \beta}\left(\left.\widetilde{V}_{1}\right|_{\tilde{H}}\right)^{\alpha}$ and $\widetilde{V}_{2}^{* \beta} \widetilde{V}_{1}^{\alpha}$ are isometries we may reduce the projection and get $\left(\left.\widetilde{V}_{2}\right|_{\tilde{H}}\right)^{* \beta}\left(\left.\widetilde{V}_{1}\right|_{\tilde{H}}\right)^{\alpha}=\left.\widetilde{V}_{2}^{* \beta} \widetilde{V}_{1}^{\alpha}\right|_{\tilde{H}}$. Hence $\tilde{H}$ is invariant under $\widetilde{V}_{2}^{* \beta} \widetilde{V}_{1}^{\alpha}$. Let us check that the minimal $\widetilde{V}_{2}^{* \beta} \widetilde{V}_{1}^{\alpha}$ invariant subspace containing $H$ is the whole $\widetilde{H}$ and so $\tilde{H}=\widetilde{H}$. By the definition of $\widetilde{H}$ and $\mathcal{D}$ it is enough to show that $\left(0,\left(I-V_{2}^{\beta} V_{2}^{* \beta}\right) V_{1}^{\alpha} h\right)$ belongs to the minimal $\widetilde{V}_{2}^{* \beta} \widetilde{V}_{1}^{\alpha}$ invariant subspace containing $H$ for any $h \in H$. By (4.4)

$$
\widetilde{V}_{2}^{\beta}\left(V_{2}^{* \beta} V_{1}^{\alpha} h,\left(I-V_{2}^{\beta} V_{2}^{* \beta}\right) V_{1}^{\alpha} h\right)=\left(V_{1}^{\alpha} h, 0\right)=\widetilde{V}_{1}^{\alpha}(h, 0) .
$$

Hence

$$
\widetilde{V}_{2}^{* \beta} \widetilde{V}_{1}^{\alpha}(h, 0)=\left(V_{2}^{* \beta} V_{1}^{\alpha} h,\left(I-V_{2}^{\beta} V_{2}^{* \beta}\right) V_{1}^{\alpha} h\right)
$$

and so

$$
\left(0,\left(I-V_{2}^{\beta} V_{2}^{* \beta}\right) V_{1}^{\alpha} h\right)=\widetilde{V}_{2}^{* \beta} \widetilde{V}_{1}^{\alpha}(h, 0)-\left(V_{2}^{* \beta} V_{1}^{\alpha} h, 0\right) \in \overline{\operatorname{span}}\left\{\widetilde{V}_{2}^{* \beta} \widetilde{V}_{1}^{\alpha} H, H\right\} .
$$


Let us show the properties of the extensions.

Recall that by Wold-Halmos theorem an isometry $V$ is a unilateral shift if and only if $\bigcap_{\gamma>0} V^{\gamma} H=\{0\}$. By (4.5) $\operatorname{ran}\left(\widetilde{V}_{1}^{\alpha}\right) \subset H$ and since the sequence $\left\{\operatorname{ran}\left(\widetilde{V}_{1}^{\alpha}\right)\right\}_{\alpha \geq 0}$ is decreasing

$$
\bigcap_{\gamma>0} \widetilde{V}_{1}^{\gamma} \widetilde{H}=\bigcap_{\gamma>\alpha} \widetilde{V}_{1}^{\gamma} \widetilde{H} \subset \bigcap_{\gamma>0} \widetilde{V}_{1}^{\gamma} H=\bigcap_{\gamma>0} V_{1}^{\gamma} H=\{0\} .
$$

The proof for $V_{2}$ follows similarly by (4.6).

The second property is equivalent to the implication: if $V_{1}^{* \alpha} V_{2}^{\beta}$ is an isometry, then $\widetilde{V}_{1}^{* \alpha} \widetilde{V}_{2}^{\beta}$ is an isometry. However, $V_{1}^{* \alpha} V_{2}^{\beta}$ is an isometry if and only if $\operatorname{ran}\left(V_{2}^{\beta}\right) \subset \operatorname{ran}\left(V_{1}^{\alpha}\right)$ and similarly for the extensions. Thus we need to show that if $\operatorname{ran}\left(V_{2}^{\beta}\right) \subset \operatorname{ran}\left(V_{1}^{\alpha}\right)$, then $\operatorname{ran}\left(\widetilde{V}_{2}^{\beta}\right) \subset \operatorname{ran}\left(\widetilde{V}_{1}^{\alpha}\right)$. Recall that $\mathcal{D} \subset H \ominus\left(\operatorname{ker} V_{1}^{* \alpha} \cap \operatorname{ker} V_{2}^{* \beta}\right)=\overline{\operatorname{span}}\left\{\operatorname{ran}\left(V_{1}^{\alpha}\right), \operatorname{ran}\left(V_{2}^{\beta}\right)\right\}$. Thus if $\operatorname{ran}\left(V_{2}^{\beta}\right) \subset$ $\operatorname{ran}\left(V_{1}^{\alpha}\right)$ then $\mathcal{D} \subset \operatorname{ran}\left(V_{1}^{\alpha}\right)$ and from (4.5), (4.6) $\operatorname{ran}\left(\widetilde{V}_{2}^{\beta}\right)=\{(h, 0): h \in$ $\left.\operatorname{ran}\left(V_{2}^{\beta}\right) \oplus \mathcal{D}\right\} \subset\left\{(h, 0): h \in \operatorname{ran}\left(V_{1}^{\alpha}\right)\right\} \subset \operatorname{ran}\left(\widetilde{V}_{1}^{\alpha}\right)$.

The third property is equivalent to the implication: if $\operatorname{ran}\left(V_{2}^{n}\right)=\operatorname{ran}$ $\left(V_{1}^{m}\right)$, then $\operatorname{ran}\left(\widetilde{V}_{2}^{n}\right)=\operatorname{ran}\left(\widetilde{V}_{1}^{m}\right)$. Denote $U=V_{2}^{* n} V_{1}^{m}$ which, as a unitary operator, by Remark 1.4 and Fuglede-Putnam theorem doubly commutes with $V_{1}, V_{2}$. Then $V_{1}^{m}=V_{2}^{n} U$ and $U \mathcal{D}=\mathcal{D}$. By (4.1) we get

$$
\begin{aligned}
\widetilde{V}_{1}^{m}(h, f) & =\left(V_{1}^{m} h+V_{2}^{* \beta} V_{1}^{m} f,\left(I-V_{2}^{\beta} V_{2}^{* \beta}\right) V_{1}^{m} f\right) \\
& =\left(V_{2}^{n} U h+V_{2}^{* \beta} V_{2}^{n} U f,\left(I-V_{2}^{\beta} V_{2}^{* \beta}\right) V_{2}^{n} U f\right)=\widetilde{V}_{2}^{n}(U h, U f) .
\end{aligned}
$$

Eventually, $U H=H$ and $U \mathcal{D}=\mathcal{D}$ implies that $\operatorname{ran}\left(\widetilde{V}_{2}^{n}\right)=\operatorname{ran}\left(\widetilde{V}_{1}^{m}\right)$.

A result similar to Proposition 4.3 but for a unitary extension follows by the properties of the isometric extension proved in Proposition 4.3.

Corollary 4.4. Let $\left(V_{1}, V_{2}\right)$ be a pair of commuting isometries such that $V_{2}^{* 2 \beta}$ $V_{1}^{2 \alpha}$ is a unitary operator. There is a minimal extension to a pair of commuting isometries $\left(\widehat{V}_{1}, \widehat{V}_{2}\right)$ such that $\widehat{V}_{2}^{* \beta} \widehat{V}_{1}^{\alpha}$ is a unitary operator.

Proof. By Proposition 4.3 there is an extension $\left(\tilde{V}_{1}, \tilde{V}_{2}\right)$ such that $\tilde{V}_{1}^{* 2 \alpha} \tilde{V}_{2}^{2 \beta}$ is unitary (it follows by the third property for $m=2 \alpha, n=2 \beta$ ) and $\tilde{V}_{1}^{* \alpha} \tilde{V}_{2}^{\beta}$ is an isometry. Thus $\tilde{V}_{2}^{* 2 \beta} \tilde{V}_{1}^{2 \alpha}$ is unitary and $\tilde{V}_{2}^{* \beta} \tilde{V}_{1}^{\alpha}$ is a co-isometry. By Proposition 4.3 (the second property) $\left(\tilde{V}_{1}, \tilde{V}_{2}\right)$ can be extended to $\left(\widehat{V}_{1}, \widehat{V}_{2}\right)$ such that $\widehat{V}_{2}^{* \beta} \widehat{V}_{1}^{\alpha}$ is a unitary operator. Obviously the extension is minimal as both steps provide a minimal extension by Proposition 4.3.

Now we are ready to show that any pair satisfying $(\dagger)$ may be extended to a pair of the form $(\ddagger)$.

Theorem 4.5. Any pair of commuting isometries $\left(V_{1}, V_{2}\right)$ on a Hilbert space $H$ satisfying

$$
V_{1}^{m}=U V_{2}^{n}
$$

for a unitary operator $U$ commuting with $V_{1}, V_{2}$ and relatively prime, positive integers $m, n$ may be extended to a pair

$$
\left(\tilde{U}^{k} \tilde{V}^{n}, \tilde{U}^{l} \tilde{V}^{m}\right)
$$


where:

- $\tilde{U}$ is a unitary operator commuting with an isometry $\tilde{V}$,

- $H \in \operatorname{Lat}\left(\tilde{V}^{m}, \tilde{V}^{n}\right)$ and

- $(k, l)$ are unique integers such that $0<k<n, 0 \leq l<m$ and $k m-\ln =1$.

Moreover, the extension may be chosen to be minimal, and for a minimal extension if $V_{1}, V_{2}$ are unilateral shifts, then $\tilde{V}$ is a unilateral shift.

Proof. Assume that $\left(V_{1}, V_{2}\right)$ extends to a commuting pair of isometries $\left(\tilde{V}_{1}\right.$, $\left.\tilde{V}_{2}\right)$ such that $\tilde{V}_{1}^{* l} \tilde{V}_{2}^{k}$ is an isometry and $\tilde{V}_{2}^{* n} \tilde{V}_{1}^{m}$ is unitary. By Remark 4.1 $\tilde{V}_{1}=\tilde{U}^{k} \tilde{V}^{m}, \tilde{V}_{2}=\tilde{U}^{l} \tilde{V}^{n}$ where $\tilde{U}=\tilde{V}_{2}^{* n} \tilde{V}_{1}^{m}$ and $\tilde{V}=\tilde{V}_{1}^{* l} \tilde{V}_{2}^{k}$. We need to show that $H \in \operatorname{Lat}\left(\tilde{V}^{m}, \tilde{V}^{n}\right)$. Since $\tilde{V}_{2}$ is an extension of $V_{2}$ then $U=$ $V_{2}^{* n} V_{1}^{m}=\left.P_{H} \tilde{V}_{2}^{* n} \tilde{V}_{1}^{m}\right|_{H}=\left.P_{H} \tilde{U}\right|_{H}$ and similarly $U^{*}=\left.P_{H} \tilde{U}^{*}\right|_{H}$. Since both $U$ and $\tilde{U}^{*}$ are unitary, we may reduce the projection. Thus $\left.\tilde{U}\right|_{H}=U$ and $\left.\tilde{U}^{*}\right|_{H}=U^{*}$ and so $H$ reduces $\tilde{U}$. Hence and since $H \in \operatorname{Lat}\left(\tilde{V}_{1}, \tilde{V}_{2}\right)$ we get $H \in \operatorname{Lat}\left(\tilde{V}^{m}, \tilde{V}^{n}\right)$.

It remains to prove that $\left(V_{1}, V_{2}\right)$ may be extended to a commuting pair of isometries $\left(\tilde{V}_{1}, \tilde{V}_{2}\right)$ such that $\tilde{V}_{1}^{* l} \tilde{V}_{2}^{k}$ is an isometry and $\tilde{V}_{2}^{* n} \tilde{V}_{1}^{m}$ is unitary. The integers $k, l$ exist and are unique by Remark 1.5. For any positive integer $q$ let

$$
q k=p n+r
$$

where $p, r$ denote the quotient and the remainder of $q k / n$, respectively. On the other hand, $k m=l n+1$. Hence $p m n+r m=(p n+r) m=q k m=$ $q(\ln +1)=q \ln +q$. Dividing $p m n+r m=q l n+q$ by $n$ we get

$$
q l=p m-\rho
$$

where $\rho:=\frac{q}{n}-\frac{r}{n} m$. Thus $\rho=p m-q l \in \mathbb{Z}$ and since $r$, as a reminder of division by $n$, is less than $n$, we get $\rho>0$ for $q \geq m n$. The assumption $V_{1}^{m}=U V_{2}^{n}$ implies $U^{*}=V_{1}^{* m} V_{2}^{n}$, and by Remark $1.4 U^{* p}=V_{1}^{* p m} V_{2}^{p n}$. Hence $V_{1}^{* q l} V_{2}^{q k}=V_{1}^{* p m} V_{1}^{\rho} V_{2}^{p n} V_{2}^{r}=U^{* p} V_{1}^{\rho} V_{2}^{r}$, and so $V_{1}^{* q l} V_{2}^{q k}$ is an isometry for any $q \geq m n$. Consequently, there is $i \geq 0$ minimal, such that $V_{1}^{* 2^{i} l} V_{2}^{2^{i} k}$ is an isometry. Using Proposition $4.3 i$-times we obtain a minimal extension to a pair $\tilde{V}_{1}, \tilde{V}_{2}$ such that $\tilde{V}_{1}^{* l} \tilde{V}_{2}^{k}$ is an isometry. Note that by the third property in Proposition 4.3 the operator $\tilde{V}_{2}^{* n} \tilde{V}_{1}^{m}$ is unitary since $V_{2}^{* n} V_{1}^{m}=U$ is unitary.

If $V_{1}, V_{2}$ are unilateral shifts, then by the first property in Proposition 4.3 extensions are unilateral shifts and so $\tilde{V}$ is a unilateral shift as well.

Since the proof of Theorem 4.5 is based on Proposition 4.3, the extension preserves all the properties described in Proposition 4.3. As a conclusion of Proposition 3.5 and Theorem 4.5 we get the extension of an arbitrary pair of commuting isometries.

Theorem 4.6. Any pair of commuting isometries $\left(V_{1}, V_{2}\right)$, for any relatively prime, positive integers $m, n$ may be extended to a pair $\left(\widehat{U}^{k} \widehat{V}^{n}, \widehat{U}^{l} \widehat{V}^{m}\right)$ where $\widehat{U}$ is a unitary operator commuting with an isometry $\widehat{V}$ and $(k, l)$ are unique integers such that $0<k<n, 0 \leq l<m$ and $k m-l n=1$. Moreover, the extension may be chosen minimal. 


\section{Applications}

By Theorem 4.6, any pair of commuting isometries may be extended to a pair of the form $(\ddagger)$. Hence an arbitrary pair is of the form $\left(\left.U^{l} V^{n}\right|_{H},\left.U^{l} V^{m}\right|_{H}\right)$ where $H \in \operatorname{Lat}\left(U^{k} V^{n}, U^{l} V^{m}\right)$. Since the model for pairs of the form $\left(U^{l} V^{n}\right.$, $\left.U^{l} V^{m}\right)$ is given by Remark 4.2, a description of Lat $\left(U^{k} V^{n}, U^{l} V^{m}\right)$ provides a model for an arbitrary pair of commuting isometries. Unfortunately $\operatorname{Lat}\left(U^{k} V^{n}, U^{l} V^{m}\right)$ is characterized only for some cases like in Theorem 2.7. We show applications of this case and investigate $\operatorname{Lat}\left(U^{k} V^{n}, U^{l} V^{m}\right)$ more generally.

Let a pair of commuting isometries $\left(V_{1}, V_{2}\right)$ satisfy $V_{1}^{m}=U V_{2}^{n}$ where $U$ is a unitary operator commuting with $V_{1}, V_{2}$. By Remark 3.2 such a pair decomposes between a pair of unitary operators and a pair of unilateral shifts. In fact, any pair of unitary operators $\left(U_{1}, U_{2}\right)$ satisfies the equality $U_{1}^{m}=$ $U U_{2}^{n}$ where $U=U_{2}^{* n} U_{1}^{m}$. We focus on pairs of unilateral shifts satisfying $V_{1}^{m}=U V_{2}^{n}$. If $U$ has an eigenvalue $\lambda$, then the corresponding eigenspace $H_{\lambda}$ is reducing under $V_{1}, V_{2}$ by commutativity of $U$ with $V_{1}, V_{2}$. Moreover, $\left.V_{1}^{m}\right|_{H_{\lambda}}=\left.\lambda V_{2}^{n}\right|_{H_{\lambda}}$. Invariant subspaces of such pairs are described by the following corollary of Theorem 4.5.

Corollary 5.1. Let a pair of commuting unilateral shifts $\left(V_{1}, V_{2}\right)$ on a Hilbert space $H$ satisfy $V_{1}^{m}=\lambda V_{2}^{n}$ for relatively prime, positive integers $m, n$ and a complex number $\lambda$. Then there is a unilateral shift $\tilde{V} \in \mathcal{B}(\tilde{H})$ such that $H \subset \tilde{H}$ and $\operatorname{Lat}\left(V_{1}, V_{2}\right)=\left\{H \cap \mathcal{N}: \mathcal{N} \in \operatorname{Lat}\left(\tilde{V}^{m}, \tilde{V}^{m}\right)\right\}$ where $\operatorname{Lat}\left(\tilde{V}^{n}, \tilde{V}^{m}\right)$ is described by Theorem $2 . \%$.

In the general case a similar but weaker result follows from Theorem 4.6. The result is weaker since there is no description similar to Theorem 2.7. We assume a general pair of commuting isometries. However, even if we assume a pair of unilateral shifts, an extension may be a general pair of isometries, recall Example 3.6.

Corollary 5.2. For a commuting pair of isometries $\left(V_{1}, V_{2}\right)$ on a Hilbert space $H$ and positive, relatively prime integers $m, n$ there are an isometry $\widehat{V}$ commuting with a unitary operator $\widehat{U}$ on a Hilbert space $\tilde{H}$ such that

$$
V_{1}=\left.\widehat{U}^{k} \widehat{V}^{n}\right|_{H}, V_{2}=\left.\widehat{U}^{l} \widehat{V}^{m}\right|_{H} \text { and } H \in \operatorname{Lat}\left(\widehat{U}^{k} \widehat{V}^{m}, \widehat{U}^{l} \widehat{V}^{n}\right)
$$

where the integers $0<k<m, 0 \leq l<n$ are such that $k m-l n=1$. Moreover, $\operatorname{Lat}\left(V_{1}, V_{2}\right)=\left\{H \cap \mathcal{N}: \mathcal{N} \in \operatorname{Lat}\left(\widehat{U}^{k} \widehat{V}^{n}, \widehat{U}^{l} \widehat{V}^{m}\right)\right\}$.

Let us focus on $\operatorname{Lat}\left(U^{k} V^{n}, U^{l} V^{m}\right)$. Since Theorem 4.6 provides the extension for any relatively prime, positive integers $m, n$, we may assume $m=n=1$ and so $k=1, l=0$. In other words, we make an approach to a description of $\operatorname{Lat}(U V, V)$ where $U$ is a unitary operator commuting with a unilateral shift $V$. The advantage is that $U V, V$ are unitarily equivalent. The following remark describes the lattice of subspaces invariant under a pair of unitarily equivalent isometries. 
Remark 5.3. Let $V, W \in \mathcal{B}(H)$ where $V$ is an isometry and $W$ is a unitary operator (not commuting with $V$ ). Then

$$
\operatorname{Lat}\left(W V W^{*}, V\right)=\operatorname{Lat}(V) \cap W \operatorname{Lat}(V)
$$

where $W \operatorname{Lat}(V)=\{W \mathcal{M}: \mathcal{M} \in \operatorname{Lat}(V)\}$. Indeed, it is an easy observation that $\mathcal{M}$ is invariant under $W V W^{*}$ if and only if $W^{*} \mathcal{M}$ is invariant under $V$. Since $W$ is unitary, $\left\{\mathcal{M}: W^{*} \mathcal{M} \in \operatorname{Lat}(V)\right\}=W \operatorname{Lat}(V)$.

Remark 5.3 is shown in the context of the pair $(U V, V)$. However, isometries $U^{k} V^{n}, U^{l} V^{m}$ are unilateral shifts of multiplicities $m \operatorname{dim} \operatorname{ker} V^{*}$ and $n \operatorname{dim} \operatorname{ker} V^{*}$ respectively, so are unitarily equivalent if $\operatorname{dim} \operatorname{ker} V^{*}=$ $\infty$. Hence Remark 5.3 has wider applications. We continue investigating Lat $(U V, V)$. Assuming $V$ to be a unilateral shift, the model for the pair $(U V, V)$ given by Remark 4.2 is $\left(T_{z} \otimes \mathcal{U}, T_{z} \otimes I\right)$ on $H^{2}(\mathbb{T}) \otimes \mathcal{H}$. Recall that $P_{i} \in \mathcal{B}\left(H^{2}(\mathbb{T})\right)$ denotes the projection onto the space $\mathbb{C} z^{i}$.

Theorem 5.4. Let $V=T_{z} \otimes I, U=I \otimes \mathcal{U}$ and $W=\sum_{i>0} P_{i} \otimes \mathcal{U}^{i}$ operate on $H^{2}(\mathbb{T}) \otimes \mathcal{H}$. The subspaces jointly invariant under $(U V, V)$ are precisely those of the form $\hat{\theta}\left(H^{2}(\mathbb{T}) \otimes \mathcal{H}\right)$ where $\hat{\theta}$ is an inner function satisfying

$$
\hat{\theta}\left(H^{2}(\mathbb{T}) \otimes \mathcal{H}\right)=W \hat{\eta}\left(H^{2}(\mathbb{T}) \otimes \mathcal{H}\right)
$$

for some inner function $\hat{\eta}$.

Proof. Note that $W$ is a unitary operator not commuting with $V$. Let $\hat{\eta}$ be an inner function. Then $W \hat{\eta}(z)$ is a partial isometry for almost every $z$ with the same initial space as the initial space of $\hat{\eta}(z)$, so the same initial space for almost every $z$. However, $W \hat{\eta}$ may not commute with $V$ and so it may not be an inner function. On the other hand, if $W \hat{\eta}\left(H^{2}(\mathbb{T}) \otimes \mathcal{H}\right)=\hat{\theta}\left(H^{2}(\mathbb{T}) \otimes \mathcal{H}\right)$ for $\hat{\theta}$ an inner function then $\hat{\theta}\left(H^{2}(\mathbb{T}) \otimes \mathcal{H}\right) \in \operatorname{Lat}(V) \cap W \operatorname{Lat}(V)$. Moreover, there are no other subspaces in $\operatorname{Lat}(V) \cap W \operatorname{Lat}(V)$. Hence the theorem follows by Remark 5.3 provided we show $U V=W V W^{*}$. Check that

$$
W V W^{*}=\left(\sum_{j \geq 0} P_{j} \otimes \mathcal{U}^{j}\right)\left(T_{z} \otimes I\right)\left(\sum_{i \geq 0} P_{i} \otimes \mathcal{U}^{* i}\right)=\sum_{i \geq 0} \sum_{j \geq 0} P_{j} T_{z} P_{i} \otimes \mathcal{U}^{j} \mathcal{U}^{* i} .
$$

On the other hand, $T_{z} P_{i} H^{2}(\mathbb{T})=P_{i+1} H^{2}(\mathbb{T})$ yields $P_{j} T_{z} P_{i}=0$ for $j \neq i+1$ and $P_{i+1} T_{z} P_{i}=T_{z} P_{i}$. Thus

$$
\begin{aligned}
\sum_{i \geq 0} \sum_{j \geq 0} P_{j} T_{z} P_{i} \otimes \mathcal{U}^{j} \mathcal{U}^{* i} & =\sum_{i \geq 0} T_{z} P_{i} \otimes \mathcal{U} \\
& =\left(T_{z} \otimes \mathcal{U}\right)\left(\sum_{i \geq 0} P_{i} \otimes I\right)=T_{z} \otimes \mathcal{U}=U V .
\end{aligned}
$$
model.

As a corollary of Theorem 5.4 and Theorem 4.6, we get the following

Theorem 5.5. Any pair of commuting isometries have a minimal extension to a pair $(U V, V)$ where $U$ is a unitary operator commuting with an isometry $V$. Those of them for which $V$ is a unilateral shift are of the form 


$$
\left(\left.\left(T_{z} \otimes I\right)\right|_{\mathcal{M}},\left.\left(T_{z} \otimes \mathcal{U}\right)\right|_{\mathcal{M}}\right)
$$

where

- $\mathcal{U} \in \mathcal{B}(\mathcal{H})$ is a unitary operator,

- $\mathcal{M}=\hat{\theta}\left(H^{2}(\mathbb{T}) \otimes \mathcal{H}\right)$ where $\hat{\theta}$ is an inner function satisfying

$$
\hat{\theta}\left(H^{2}(\mathbb{T}) \otimes \mathcal{H}\right)=W \hat{\eta}\left(H^{2}(\mathbb{T}) \otimes \mathcal{H}\right)
$$

for some inner function $\hat{\eta}$ and $W=\sum_{i \geq 0} P_{i} \otimes \mathcal{U}^{i}$.

According to Example 3.6 the theorem above does not give a universal model even for pairs of unilateral shifts. However, it describes interesting examples, like operators of multiplication by independent variables on the Hardy space over the torus and their restrictions to invariant subspaces. We finish by a detailed investigation of this example. According to Example 3.7 the subspace $\mathcal{H}$ described in the model in Theorem 5.5 equals $L^{2}(\mathbb{T})$. Hence we get operators on $H^{2}(\mathbb{T}) \otimes L^{2}(\mathbb{T})$ which, via a unitary equivalence, may be considered as a subspace of $L^{2}\left(\mathbb{T}^{2}\right)$.

Theorem 5.6. Let $\left(L_{w}, L_{z}\right)$ be operators of multiplication by independent variables on $L^{2}\left(\mathbb{T}^{2}\right)$. Set $\Delta^{2}\left(\mathbb{T}^{2}\right):=\left\{f \in L^{2}\left(\mathbb{T}^{2}\right): \hat{f}_{i, j}=0\right.$ for $\left.j<-i\right\}$ and $W \in \mathcal{B}\left(\Delta^{2}\left(\mathbb{T}^{2}\right)\right)$ defined by $W w^{i} z^{j}=w^{2 i+j} z^{-i}$. Then

$$
\operatorname{Lat}\left(\left.L_{w}\right|_{\Delta^{2}\left(\mathbb{T}^{2}\right)},\left.L_{z}\right|_{\Delta^{2}\left(\mathbb{T}^{2}\right)}\right)=\operatorname{Lat}\left(\left.L_{w}\right|_{\Delta^{2}\left(\mathbb{T}^{2}\right)}\right) \cap W \operatorname{Lat}\left(\left.L_{w}\right|_{\Delta^{2}\left(\mathbb{T}^{2}\right)}\right) .
$$

Proof. Note that $L_{w}=L_{w \bar{z}} L_{z}$ and the subspace $\Delta^{2}\left(\mathbb{T}^{2}\right)$ is reducing under $L_{w \bar{z}}$ and invariant under $L_{w}, L_{z}$. The model for $\left.L_{z}\right|_{\Delta^{2}\left(\mathbb{T}^{2}\right)}$ yields $\Delta^{2}\left(\mathbb{T}^{2}\right) \simeq$ $H^{2}(\mathbb{T}) \otimes \mathcal{H}$ and $\left.L_{z}\right|_{\Delta^{2}\left(\mathbb{T}^{2}\right)} \simeq T_{v} \otimes I$ where

$$
\mathcal{H}=\operatorname{ker}\left(\left.L_{z}\right|_{\Delta^{2}\left(\mathbb{T}^{2}\right)}\right)^{*}=\operatorname{ker}\left(\left.L_{w}\right|_{\Delta^{2}\left(\mathbb{T}^{2}\right)}\right)^{*}=\overline{\operatorname{span}}\left\{w^{i} z^{-i}: i \in \mathbb{Z}\right\}
$$

and $v$ is the variable in $\mathbb{T}$ while $(w, z)$ remains assigned to $\mathbb{T}^{2}$. Moreover, $L_{z}^{\iota}\left(\operatorname{ker}\left(\left.L_{z}\right|_{\Delta^{2}\left(\mathbb{T}^{2}\right)}\right)^{*}\right) \simeq\left(T_{v} \otimes I\right)^{\iota}(1 \otimes \mathcal{H})=v^{\iota} \otimes \mathcal{H}$ and so

$$
\Delta^{2}\left(\mathbb{T}^{2}\right) \ni w^{i} z^{j}=w^{i} z^{-i+(i+j)}=L_{z}^{i+j} w^{i} z^{-i} \simeq v^{i+j} \otimes w^{i} z^{-i} \in H^{2}(\mathbb{T}) \otimes \mathcal{H} .
$$

One can check that $\mathcal{H}$ reduces $L_{w \bar{z}}$ and $\left.\left.L_{w \bar{z}}\right|_{\Delta^{2}\left(\mathbb{T}^{2}\right)} \simeq I \otimes L_{w \bar{z}}\right|_{\mathcal{H}}$ and $\left.L_{w}\right|_{\Delta^{2}\left(\mathbb{T}^{2}\right)}$ $\left.\simeq T_{v} \otimes L_{w \bar{z}}\right|_{\mathcal{H}}$. Then by Theorem $\left.5.4 L_{w}\right|_{\Delta^{2}\left(\mathbb{T}^{2}\right)}=\left.W L_{z}\right|_{\Delta^{2}\left(\mathbb{T}^{2}\right)} W^{*}$ where $W \simeq \sum_{n \geq 0} P_{n} \otimes\left(\left.L_{w \bar{z}}\right|_{\mathcal{H}}\right)^{n}$. Hence

$$
\begin{aligned}
W w^{i} z^{j} & \simeq \sum_{n \geq 0} P_{n} \otimes\left(L_{w \bar{z}} \mid \mathcal{H}\right)^{n}\left(v^{i+j} \otimes w^{i} z^{-i}\right)=\sum_{n \geq 0}\left(P_{n} v^{i+j} \otimes w^{i+n} z^{-i-n}\right) \\
& =v^{i+j} \otimes w^{2 i+j} z^{-2 i-j} \simeq w^{2 i+j} z^{-i}
\end{aligned}
$$

Remark 5.3 finishes the proof.

\section{Acknowledgements}

The author thanks Professor Marek Słociński for a fruitful discussion which helped to prove Proposition 4.3. 
Open Access. This article is distributed under the terms of the Creative Commons Attribution 4.0 International License (http://creativecommons.org/licenses/by/4. 0/), which permits unrestricted use, distribution, and reproduction in any medium, provided you give appropriate credit to the original author(s) and the source, provide a link to the Creative Commons license, and indicate if changes were made.

Publisher's Note Springer Nature remains neutral with regard to jurisdictional claims in published maps and institutional affiliations.

\section{References}

[1] Berger, C.A., Coburn, L.A., Lebow, A.: Representation and index theory for $C^{*}$-algebras generated by commuting isometries. J. Funct. Anal. 27, 51-99 (1978)

[2] Beurling, : On two problems concerning linear transformations in Hilbert spaces. Acta. Math. 81, 239-255 (1949)

[3] Burdak, Z., Kosiek, M., Słociński, M.: Compatible pairs of commuting isometries. Linear Algebra Appl. 479, 216-259 (2015)

[4] Burdak, Z., Kosiek, M., Pagacz, P., Słociński, M.: Invariant subspaces of $\mathcal{H}^{2}\left(\mathbb{T}^{2}\right)$ and $L^{2}\left(\mathbb{T}^{2}\right)$ preserving compatibility. J. Math. Anal. Appl. 455, 17061719 (2017)

[5] Gaspar, D., Suciu, N.: On invariant subspaces on bitorus. J. Oper. Theory 30, 227-241 (1993)

[6] Halmos, P.R.: Shifts on Hilbert spaces. J. Reine Angew. Math. 208, 102-112 (1961)

[7] Izuchi, K., Nakazi, T., Seto, M.: Backward shift invariant subspaces in the bidisc. II. J. Oper. Theory 51, 361-376 (2004)

[8] Lax, P.D.: Translation invariant spaces. Acta Math. 101, 163-178 (1959)

[9] Mandrekar, V.: The validity of beurling theorems in polidiscs. Proc. Am. Math. Soc. 103, 145-148 (1988)

[10] Horák, Karel, Müller, Vladimir: Functional model for commuting isometries. Czechoslovak Math. J. 39(2), 370-379 (1989)

[11] Rudin, W.: Function Theory in Polydiscs. Benjamin, New York (1969)

[12] Radjavi, H., Rosenthal, P.: Invariant Subspaces. Springer, Berlin (1973)

[13] Sz.-Nagy, B., Foiaş, C.: Harmonic Analysis of operators on Hilbert space, North-holland publishing company, Amsterdam, London, (1970)

[14] Sarkar, J.: Wold decomposition for doubly commuting isometries. Linear Algebra Appl. 445, 289-301 (2014)

[15] Sarkar, J., Sasane, A., Wick, B.: Doubly commuting submodules of the Hardy module over polydiscs. Studia Math. 2791, 179-192 (2013)

[16] Seto, M., Yang, R.W.: Inner sequence based invariant subspaces in $H^{2}\left(D^{2}\right)$. Proc. Am. Math. Soc. 135, 2519-2526 (2007)

[17] Słociński, M.: On the Wold type decomposition of a pair of commuting isometries. Ann. Polon. Math. 37, 255-262 (1980)

[18] Wold, H.: A Study in the Analysis of Stationary Time Series. Almkvist and Wiksell, Stockholm (1954) 


\section{Zbigniew Burdak $(\bowtie)$}

Department of Applied Mathematics

University of Agriculture

ul. Balicka 253c

30-198 Kraków

Poland

e-mail: rmburdak@cyf-kr.edu.pl

Received: September 27, 2018.

Revised: March 1, 2019. 